\title{
Utilization of the van der Waals Gap of 2D Materials
}

\author{
Haifeng Que, Huaning Jiang, Xingguo Wang, Pengbo Zhai, Lingjia Meng, Peng Zhang, Yongji Gong * \\ School of Materials Science and Engineering, Beihang University, Beijing 100191, China.
}

\begin{abstract}
Since their discovery, two-dimensional (2D) materials have attracted significant research attention owing to their excellent and controllable physical and chemical properties. These materials have emerged rapidly as important material system owing to their unique properties such as electricity, optics, quantum properties, and catalytic properties. 2D materials are mostly bonded by strong ionic or covalent bonds within the layers, and the layers are stacked together by van der Waals forces, thereby making it possible to peel off 2D materials with few or single layers. The weak interaction between the layers of $2 \mathrm{D}$ materials also enables the use of van der Waals gaps for regulating the electronic structure of the system and further optimizing the material properties. The introduction of guest atoms can significantly change the interlayer spacing of the original material and coupling strength between the layers. Also, interaction between the guest and host atom also has the potential to change the electronic structure of the original material, thereby affecting the material properties. For example, the electron structure of a host can be modified by interlayer guest atoms, and characteristics such as carrier concentration, optical transmittance, conductivity, and band gap can be tuned. Organic cations intercalated between the layers of 2D materials can produce stable superlattices, which have great potential for developing new electronic and optoelectronic devices. This method enables the modulation of the electrical, magnetic, and optical properties of the original materials, thereby establishing a family of 2D materials with widely adjustable electrical and optical properties. It is also possible to introduce some new properties to the $2 \mathrm{D}$ materials, such as magnetic properties and catalytic properties, by the intercalation of guest atoms. Interlayer storage, represented by lithium-ion batteries, is also an important application of 2D van der Waals gap utilization in energy storage, which has also attracted significant research attention. Herein, we review the studies conducted in recent years from the following aspects: (1) changing the layer spacing to change the interlayer coupling; (2) introducing the interaction between guest and host atoms to change the physico-chemical properties of raw materials; (3) introducing the guest substances to obtain new properties; and (4) interlayer energy storage. We systematically describe various interlayer optimization methods of 2D van der Waals gaps and their effects on the physical and chemical properties of synthetic materials, and suggest the direction of further development and utilization of 2D van der Waals gaps.
\end{abstract}

Key Words: Two-dimensional materials; van der Waals gap; Intercalation technology; Interlayer interaction; Performance optimization; Energy storage

\footnotetext{
Received: October 23, 2020; Revised: November 21, 2020; Accepted: November 23, 2020; Published online: December 1, 2020.

*Corresponding author. Email: yongjigong@buaa.edu.cn; Tel.: +86-18513835303.

The project was supported by the National Natural Science Foundation of China (51872012) and the Key Technologies Research and Development Program of China (2018YFA0306900). 


\section{二维材料范德华间隙的利用}

阙海峰, 江华宁, 王兴国, 翟朋博, 孟令佳, 张鹏, 宫勇吉* 北京航空航天大学材料科学与工程学院, 北京 100191

摘要: 二维材料因为其优异且可调的各种物理化学性质自被发现以来就引起了科研工作者的极大关注。其电学、光学、 量子、催化等方面的一些独特性质使其迅速成为一类极其重要的材料体系。二维材料层间弱结合的性质为利用范德华间 隙来调控体系的电子结构从而进一步优化材料性能创造了条件。客体原子的引入可以显著改变原有材料的层间间距, 改 变层间的耦合强度。客体与宿主原子的相互作用也可能改变原始材料的电子结构, 从而影响材料的多方面性能, 甚至带 来新的性质。以锂离子电池为代表的层间存储也是二维范德华间隙在能源存储方面的重要应用, 一直受到许多科研工作 者的关注。在本综述中, 我们从利用插层法改变层间距从而改变层间耦合, 引入客体物质与宿主原子相互作用从而改变 原材料的物理化学性质或引入新性质和层间储能四个方面系统化阐述了二维材料范德华间隙的各种调控方法及其对合 成材料的物理、化学性能的巨大影响, 并展望了二维范德华间隙进一步开发利用的方向。

关键词: 二维材料; 范德华间隙; 插层技术; 层间相互作用; 性能优化; 能源存储 中图分类号: 0646

\section{1 引言}

自从石墨烯被发现以来, 二维材料就因为其 独特的物理化学性质成为研究热点 ${ }^{1-3}$ 。其电学、光 学、量子、催化等独特且可调谐的特性使得二维材 料迅速成为一类极其重要的材料体系, 其中一些独 特的二维材料甚至具有可调的超导性和磁性 4,5 。二 维材料面内多以强的离子键或共价键成键, 层间 通过层与层之间的范德华力堆积在一起, 这种结 构也使得剥离少层或单层的二维材料成为可能。石 墨烯的成功剥离, 为我们正式开启了二维材料世 界的大门。

不同于传统材料, 二维材料中范德华间隙的存 在提供了调控其性质或人工创造新材料的手段。 二维材料层间弱结合的特性使得其层间易于掺杂 外来分子、离子、原子等客体。例如二维TMDs (过 渡金属硫族化物)随着插入不同组分和浓度的插 入剂, 其本征电子结构可以被有效调控, 体现出不 同的电输运, 光学和磁学特性 6 。范德华间隙中插 入剂的引入, 可以显著改变宿主材料的层间距, 调 控层与层之间的范德华耦合作用, 从而优化二维

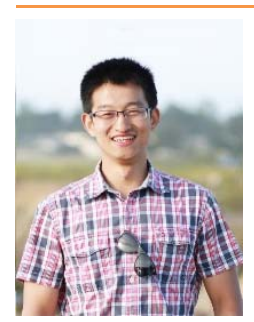

宫勇吉, 2011年本科毕业于北京大学 化学与分子工程学院。2015年博士毕 业于美国莱斯大学。2016-2017年在美 国斯坦福大学从事博士后研究。现为 北京航空航天大学材料科学与工程学 院教授, 博士生导师。主要研究方向为 二维材料的合成、性质调控及其在信息器件、新能源器件 方面的应用。
材料的物理、化学性能。暴露的层间位点被证明能 够改变材料的催化特性, 也可用来稳定纳米团簇 乃至单原子, 有望开发出一类新型的高效及高原 子利用率的催化剂。二维范德华间隙中客体物种 与宿主存在着各种各样的相互作用, 其相互作用 过程及机理也吸引着一大批科研工作者的注意 力, 选用不同种类的客体物种会显著影响插层过 程和所得到材料的电子性质, 引起材料性能的改 变, 甚至出现原来所不具有的新性能。此外, 二维 材料的层间存储也正在成为能源领域越来越重要 的发展方向, 受到国内外众多科研工作者的关注。 在本综述中, 我们将从改变二维材料的层间距以 改变层间耦合、客体原子与二维材料相互作用、客

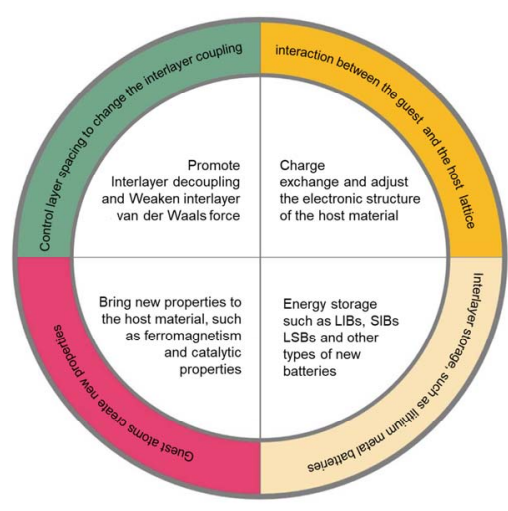

图 1 四个主要分支

Fig. 1 Four main branches of the article.

(a) Control layer spacing to change the interlayer coupling;

(b) Interaction between the guest and the host lattice; (c) Guest atoms create new properties; (d) Interlayer storage, such as lithium metal batteries. 
体原子带来新性质和层间存储四个主要方面系统 化阐述近年来的各项重要工作(图1), 着重介绍二 维材料范德华间隙的各种层间调控方法及其对合 成材料的物理、化学的巨大影响, 并对未来二维范 德华间隙的进一步开发利用做简要阐述。

\section{2 改变层间距以改变层间耦合}

层与层之间的相互作用(即层间耦合)显然对 二维材料的性质起到了至关重要的作用。将客体 物种插入二维材料的范德瓦尔斯间隙, 已被证明 可以在大块晶体中诱导出类超薄纳米片的特性, 有 效地改变原有材料的电子性质 ${ }^{7-9}$ 。例如一些具有 层数依赖性的二维材料 (如 $\mathrm{MoS}_{2}$ ) 在多层时具有间 接带隙, 其单层纳米片具有直接带隙 ${ }^{10}$, 而当层间 距扩大或层间耦合减弱达到一定比例时, 相邻层 就会发生解耦, 表现出类似单层纳米片的直接带 隙性质 ${ }^{9-15}$ 。层间距的扩大会显著减弱二维材料层 间的范德华作用, 从而调控其带隙、电导率、热电 性能、光学性能、磁性和乃至超导性 ${ }^{16-21}$, 对于探 究扩展二维材料新奇的物理、化学特性有着深远 的意义。

在调控二维材料的层间距方面, 科研工作者 们近年来发现了一系列有效手段。通过杂原子掺 杂和利用金属离子, 小分子, 金属原子, 有机分子 等进行插层都可以有效改变二维材料的层间距。 杂原子掺杂在改变层间耦合的同时通常会引起与 基体材料的电荷转移或其他相互作用 ${ }^{22-24}$, 重原子 的存在也会增加自旋轨道耦合, 引起超导性质, 热 电性能或自旋极化的改变 ${ }^{25-29}$ 。Xie等人报道了通 过水热法成功合成了 $\mathrm{O}$ 掺杂的 $\mathrm{MoS}_{2}$ 纳米薄片, 掺 杂后和掺杂前的 $\mathrm{MoS}_{2}$ 模型分别如图 $2 \mathrm{a}, \mathrm{b}$ 所示。图 $2 \mathrm{c}, \mathrm{d}$ 的XRD和透射电镜图像也证实了层间间距的 扩大 ${ }^{30}$ 。Qin等人通过溶胶-凝胶路线实现了增大层 间距的 $\mathrm{N}$ 掺杂 $\mathrm{MoS}_{2}$, 其层间间距由 $0.62 \mathrm{~nm}$ 扩大到 $0.65 \mathrm{~nm}^{31}$ 。 $\mathrm{Ye}$ 等人也通过简单的水热反应证实, $\mathrm{P}$ 掺杂可以调节 $\mathrm{MoS}_{2}$ 的层间距和电输运性质, $\mathrm{P}$ 原 子的引入抑制了 $\mathrm{MoS}_{2}(002)$ 平面的堆积, 并增大了 其层间间距 ${ }^{32}$ 。HRTEM图像证实了层间距离扩大, EDX (能量色散X射线荧光光谱, 如图3a)也表明P 原子的成功掺杂。不同于氧等原子, $\mathrm{P}$ 原子更倾向 于插入层间而不是取代主晶格中的 $\mathrm{S}$ 原子。图3中 $\mathrm{b}-\mathrm{d}$ 为 $\mathrm{P}$ 原子插入后体系的电子结构和能带计算。 归因于 $\mathrm{P}$ 原子插入到 $\mathrm{MoS}_{2}$ 层间引入了额外的电 子, 随着 $\mathrm{P}$ 插入量的增加, 费米能级向导带靠拢, $\mathrm{MoS}_{2}$ 的 $p$ 型电导率变弱, 导通型出现了由 $p$ 型向 $n$ 型 的转变, 产生了 $n$ 型电导率, 显著改变了 $\mathrm{MoS}_{2}$ 的电

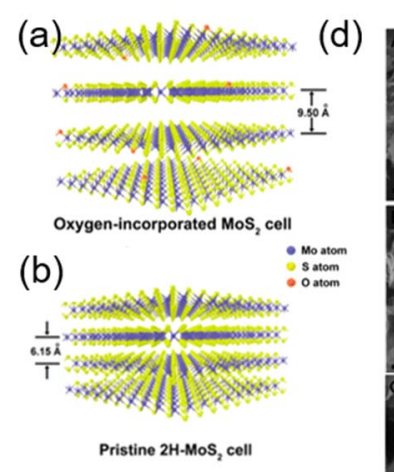

(c)
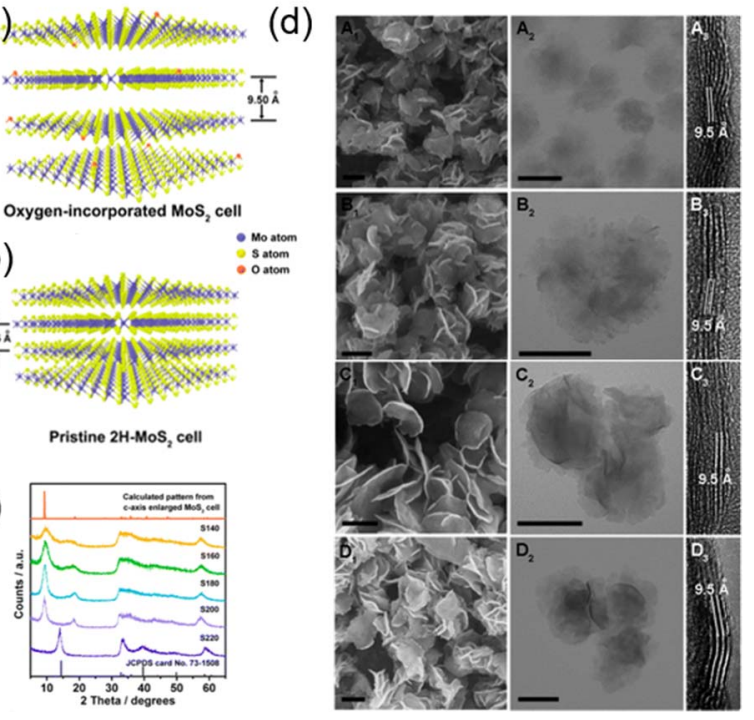

图 $2 \mathrm{MoS}_{2}$ 层间距改变的相关研究 ${ }^{30}$

Fig. 2 Studies on the change of interlayer spacing of $\mathrm{MoS}_{2}{ }^{30}$.

(a) Structural models of the oxygen-incorporated $\mathrm{MoS}_{2}$ with enlarged interlayer spacing and (b) the pristine $2 \mathrm{H}-\mathrm{MoS}_{2}$. (c) XRD patterns of the products obtained at various temperatures. (d) FE-SEM images (A1-D1). TEM images (A2-D2) and cross-sectional TEM images (A3-D3) of the oxygen-incorporated $\mathrm{MoS}_{2}$ ultrathin nanosheets synthesized at various

temperatures. Adapted from ACS Publications publisher.

输运性能。调控二维材料的层间距对于提高材料 的实际应用价值有着重要意义。对于二次电池来 说, 增大的层间距意味着层与层之间范德华作用 的显著减弱和层间间隙空间的增加, 同时降低离 子插入和脱出的势垒, 提高层间离子或电子的储 存和输运特性。因此, 增大层间距是提高离子电池 的电化学性能和倍率特性的有效方法。同样对于 二维材料催化体系而言, 层间距的增加会暴露出 更多的层间催化位点, 从而大大增加原有材料的 催化性能。

在二维材料层间插入其他二维单层或者有机 分子形成超晶格结构也被发现可以有效解耦多层 二维材料的层间耦合, 显著改变材料的电学和光 学性质。2014年, Lu等人通过在二硫化钼双分子 层之间插入一个二维单层, 形成了二硫化钼/ML/ 二硫化钼夹层三层结构 $\left(\mathrm{ML}=\mathrm{BN}, \mathrm{MoSe}_{2}, \mathrm{WS}_{2}\right.$, $\left.\mathrm{WSe}_{2}\right)^{9}$ 。他们对该范德华三层结构的电子性质进 行了全面的第一性原理研究, 发现BN单分子层是 最有效的解耦 $\mathrm{MoS}_{2}$ 层间范德华耦合的二维单层, 并且所得到的 “三明治” 状三层异质结可以恢复 $\mathrm{MoS}_{2}$ 单层的电子结构, 特别是单层的直接带隙特 性。研究者计算了不同插入单层的界面极化情况 
(a)
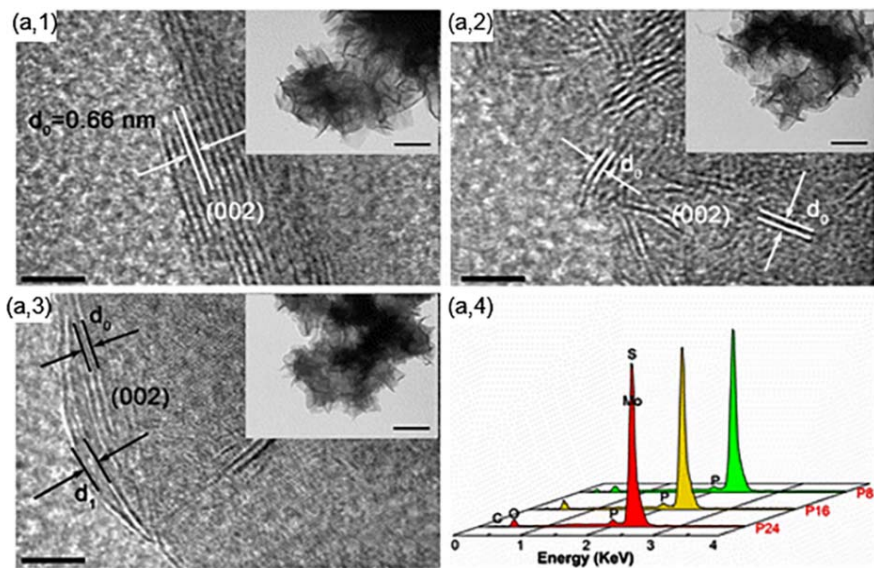

$(\mathrm{a}, 4)$

(b)

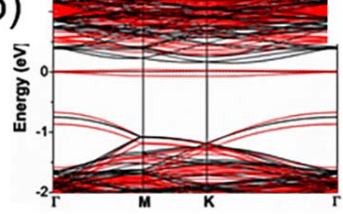

(c)

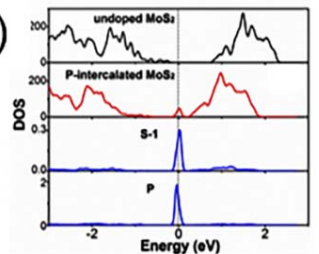

(d)

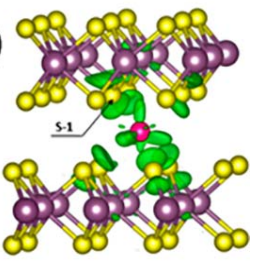

图 3 P 掺杂 $\mathrm{MoS}_{2}$ 的电输运性质和层间距研究 ${ }^{32}$

Fig. 3 Studies on interlayer spacing and electrical transport properties of P-doped $\mathrm{MoS}_{2}{ }^{32}$.

(a) HRTEM images of sample (a,1) P0, (a,2) P8, and (a,3) P24 and EDX spectra of P-doped $\mathrm{MoS}_{2}(\mathrm{a}, 4)$. (b) The calculated band structures of undoped $\mathrm{MoS}_{2}$ (black lines) and P-intercalated $\mathrm{MoS}_{2}$ (red lines). The black dashed line indicates the Fermi energy. (c) The density of states of undoped $\mathrm{MoS}_{2}$ and P-intercalated $\mathrm{MoS}_{2}$. (d) Electronic charge density difference profiles of P-intercalated $\mathrm{MoS}_{2}$. The purple, yellow, and red balls represent the Mo, S, and P atoms, respectively. Adapted from ACS Publications publisher.

(a)
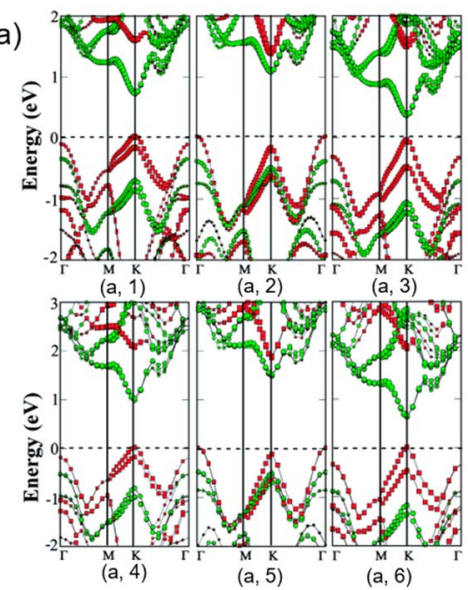

(b)
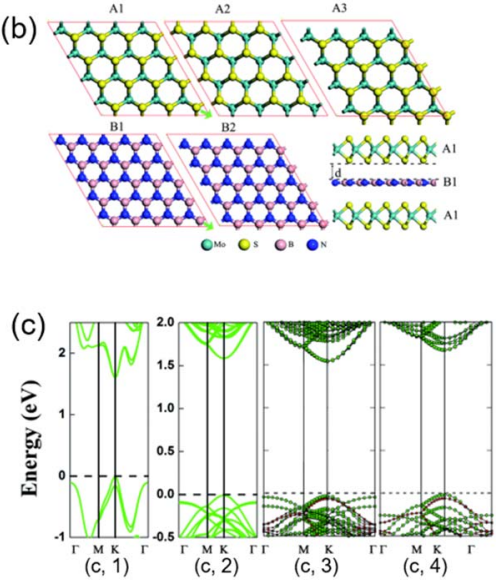

图 4 范德华三层结构电子性质的第一性原理计算研究 ${ }^{9}$

Fig. 4 First principle calculation of electronic properties of three-layer structures ${ }^{9}$.

(a) Computed band structures (PBE) of (a, 1) $\mathrm{MoS}_{2} / \mathrm{MoSe}_{2} / \mathrm{MoS}_{2}$, (a, 2) $\mathrm{MoS}_{2} / \mathrm{WS}_{2} / \mathrm{MoS}_{2}$, and (a, 3) $\mathrm{MoS}_{2} / \mathrm{WSe}_{2} / \mathrm{MoS}_{2}$ trilayer with ABA stacking, respectively. Computed band structures of (a, 4) $\mathrm{MoS}_{2} / \mathrm{MoSe}_{2}$ superlattice, (a, 5) $\mathrm{MoS}_{2} / \mathrm{WS}_{2}$ superlattice, and (a, 6) $\mathrm{MoS}_{2} / \mathrm{WSe}_{2}$ superlattice with the AB stacking, respectively. The green lines mark contribution from $\mathrm{MoS}_{2}$ layers while the red lines mark contribution from the MoSe $\mathrm{WS}_{2}$ or WSe $\mathrm{W}_{2}$ layer. (b) Top view of a $\mathrm{MoS}_{2}$ monolayer in three different supercells (marked by the red parallelogram) and a BN monolayer in two different supercells. (c) Computed electronic band structures of (c, 1) monolayer $\mathrm{MoS}_{2} ;(\mathrm{c}, 2) \mathrm{MoS}_{2} /$ vacuum layer/MoS 2 by removing the $\mathrm{BN}$ layer from the $\mathrm{MoS} / \mathrm{BN}_{2} / \mathrm{MoS}_{2}$ trilayer counterpart but with the fixed vertical location of the two $\mathrm{MoS}_{2}$ layers; (c, 3) $\mathrm{MoS}_{2} / \mathrm{BN} / \mathrm{MoS}_{2}$ with the A1B1A1 stacking; and (c, 4) a 3D superlattice of MoS $/ \mathrm{BN}$ with the

$\mathrm{AB}$ stacking. The green lines represent $\mathrm{MoS}_{2}$ layers while the red lines represent BN monolayer. Adapted from Royal Society of Chemistry publisher.

(如图4), 发现具有 $\mathrm{S} / \mathrm{S}$ 界面的 $\mathrm{MoS}_{2} / \mathrm{WS}_{2} / \mathrm{MoS}_{2}$ 三层 结构中, 相邻两层之间的电荷转移几乎为零, 与
$\mathrm{MoS}_{2} / \mathrm{MoSe}_{2} / \mathrm{MoS}_{2}$ 和 $\mathrm{MoS}_{2} / \mathrm{WSe}_{2} / \mathrm{MoS}_{2}$ 界面有显著 不同，证明了界面极化对三元层异质结构的电子 
性质有重要影响。对三维 $\mathrm{MoS}_{2} / \mathrm{BN}$ 超晶格的进一 步研究也证实了 $\mathrm{BN}$ 单层对层间相互作用解耦的 有效性。2018年, Wang等人报告了一种电化学分 子嵌入方法制备的新型稳定超晶格, 其中单层原 子与分子层交替 33 。以黑磷为例, 与十六烷基三甲 基澳化铵插层后生成的单层磷烯分子超晶格, 其 层间距离为块体黑磷的两倍以上, 有效实现了层 间的解耦, 超晶格中的磷烯分子层体现出单层的 特性, 制备的晶体管的电输运研究表明其具有超 高的迁移率和开关比。研究者利用原位检测光致 发光研究了插入过程, 原子力显微镜观察到了扩 大的厚度, XRD同样测量到了扩大的层间距(如图 5), 最终获得的单层磷烯分子超晶格具有高载流 子迁移率, 高电流开关比, 大的光学带隙和卓越的 稳定性, 为磷烯在电子和光子学领域的应用开辟
了新的道路。通过制造具有特定化学、物理、机械 和电化学性质的多功能杂化材料, 能进一步扩展 现有材料的应用范围。

从上述研究工作中, 我们可以看到调控层间 距对材料性能的显著影响。随着掺杂和插层方法 的不断发展与完善, 通过改变二维范德华间隙层 间距来调整层间耦合可能会成为提高二维材料性 能, 提升材料实际应用价值的通用策略, 也为探究 新型材料特性提供了行之有效的方法。

\section{3 客体原子与二维材料相互作用}

二维范德华间隙中引入的客体可以与宿主材 料发生相互作用, 产生各种奇异的电学、光学和热 学特性。如层间嵌入客体原子可以修饰宿主的电 子结构引起载流子浓度、光学透过率、电导率和带
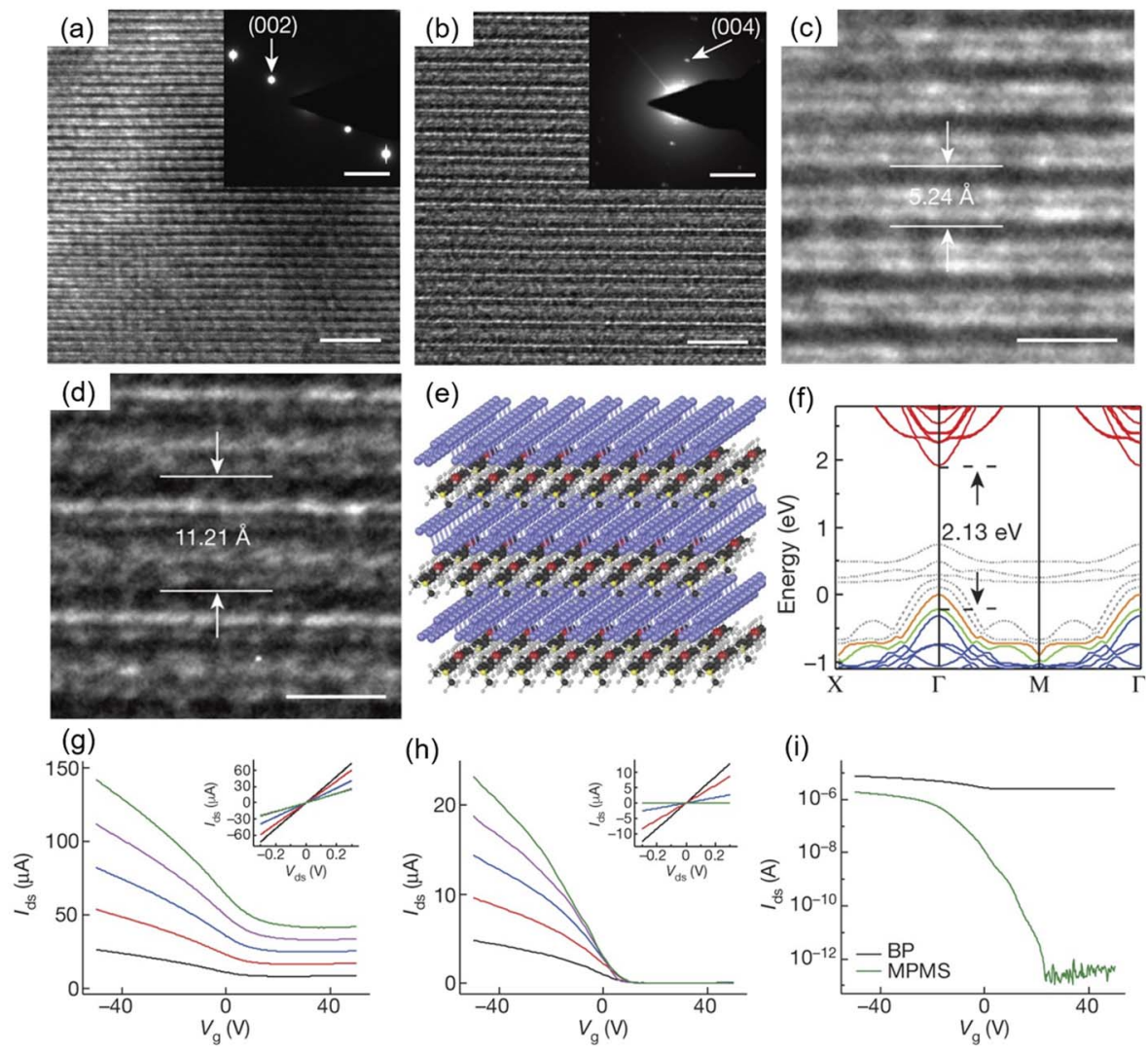

图 5 电化学分子嵌入方法制备的新型稳定超晶格的 TEM 图像能带计算和传输特性研究 ${ }^{33}$

Fig. 5 TEM images, energy band calculations and transmission characteristics of a new stable superlattice prepared by electrochemical molecular embedding method ${ }^{33}$.

(a, b) Cross sectional TEM image comparison between BP (a) and MPMS (b). Scale bars: $3 \mathrm{~nm}$. Insets are the corresponding electron diffraction pattern. Scale bars: $2 \mathrm{~nm}^{-1}$. (c, d) The corresponding high-resolution cross-sectional TEM images. Scale bars: $1 \mathrm{~nm}$. (e) Three-dimensional views of the simulated atomic structure of MPMS. (f) The simulated electronic structure of MPMS, demonstrating the enlarged bandgap of 2.13 eV in MPMS as determined by the transition between first valence-band maximum, VBM-1 (green), and conduction-band minimum, CBM (red). (g, h) Back-gate transfer characteristics of pristine BP and MPMS as the source-drain bias is stepped from $0.1 \mathrm{~V}$ (black curve) to $0.5 \mathrm{~V}$ (green curve) bias. (i) Transfer characteristics at $0.01 \mathrm{~V}$ 
隙特征的变化 ${ }^{34-39}$; 插入有机阳离子可以产生稳定 的超晶格, 在开发新型电子和光电子功能器件方 向有着巨大潜力 ${ }^{40,41}$ 。在宿主晶格的范德华间隙引 入客体为我们提供了一个新的自由度来调制原有 材料的电学、磁学和光学特性, 从而建立出电学、 光学等性质广泛可调的二维材料家族。

\section{1 插层相变}

在二维材料的范德华间隙中插入的客体往往 会与母体材料发生电荷交换, 从而引起材料物相 的变化 ${ }^{42-45}$ 。在近年来的科研工作中, 利用插层相 变来调整原有材料的电子结构被发现是一种提高 原有材料光电、催化活性的有效方法。以二硫化钼 为例, $\mathrm{MoS}_{2}$ 作为一种典型的二维过渡族硫属化物, 其单层是具有直接带隙的半导体, 在电光器件和 能源催化等领域有着巨大的应用潜力, 其热力学 稳定形式是三角棱柱形相 $\left(\mathrm{H}-\mathrm{MoS}_{2}\right)$, 其中每个钼 原子被周围的六个硫原子棱柱形配位。在 $\mathrm{Li}$ 插层 作用下, $\mathrm{MoS}_{2}$ 会经历一阶相变到亚稳态, Mo原子 变为八面体配位 $\left(1 \mathrm{~T}-\mathrm{MoS}_{2}\right)$ 。这种相变会造成状态 密度的显著变化, 使其由 $2 \mathrm{H}$ 相的半导体变为 $1 \mathrm{~T}$ 相 的金属态, 被报道具有更加优异的催化性能。

2014年, Wang等人报道了通过在三维基底上
构建边缘大量暴露的纳米结构二硫化钼颗粒, 然 后进行 $\mathrm{Li}$ 电化学插层和剥离过程, 可以获得 200 $\mathrm{mV}$ 过电位下阴极电流密度为 $200 \mathrm{~mA} \cdot \mathrm{cm}^{-2}$ 的超高 HER 性能 (见图 $6 a, b$ ) ${ }^{42}$ 。Wang 等人认为 $\mathrm{MoS}_{2}$ 的高 表面积纳米结构和 $2 \mathrm{H}$ 半导体到 $1 \mathrm{~T}$ 金属相变是其优 异的催化活性的原因。电化学稳定性试验进一步 证实了催化剂的稳定性(如图6c)。同年Wang、Shen 等人研究了 $\mathrm{MoS}_{2}$ 在电化学钠离子插层中的相变过 程, 利用电化学方法可控地插入钠离子, 并原位观 察了 $\mathrm{MoS}_{2}$ 的结构演化 ${ }^{43}$ 。2017年, Chen等人通过增 加 $\mathrm{MoS}_{2}$ 中的锂离子插层量得到了 $1 \mathrm{~T}$ 相占百分之 九十以上的二维 $\mathrm{MX}_{2}(\mathrm{M}=\mathrm{W}, \mathrm{Mo} ; \mathrm{X}=\mathrm{S}, \mathrm{Se})$ 量 子点 ${ }^{44}$ 。由于二维 $1 \mathrm{~T}-\mathrm{MoS}_{2}$ 量子点具有较高的 $1 \mathrm{~T}$ 金 属相比例和较小的横向尺寸所诱导的足够的活性 位点, 因此表现出优异的析氢反应活性(见图6d, $\mathrm{e}, \mathrm{f}$ ), 这种朝向 $2 \mathrm{D}$ 量子点的电化学路线可能有助 于在能源相关领域促进 $2 \mathrm{D}$ 材料的发展。

在插层的过程中, $2 \mathrm{H}-1 \mathrm{~T}$ 相变被认为是由于过 量的电子注入超过某一阈值 (0.29 个电子每个 $\mathrm{MoS}_{2}$ 单元), 而当选用较大的插入剂, 如钠离子 (相 对于半径较小的锂离子)和部分有机分子(如季铵 盐, 例如四庚基溴化铵)时, 由于可引入的客体原 (a)

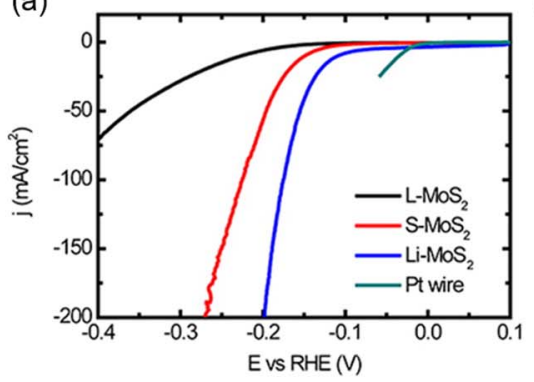

(d)

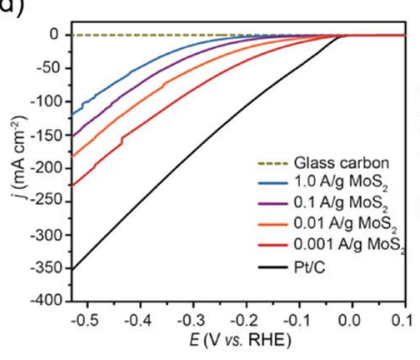

(e)

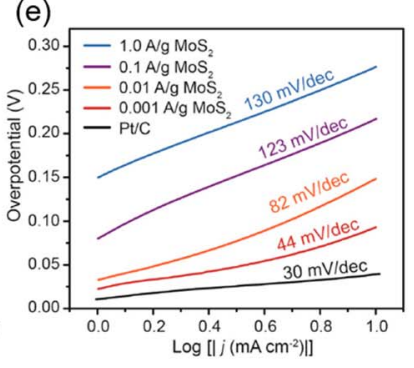

(c)
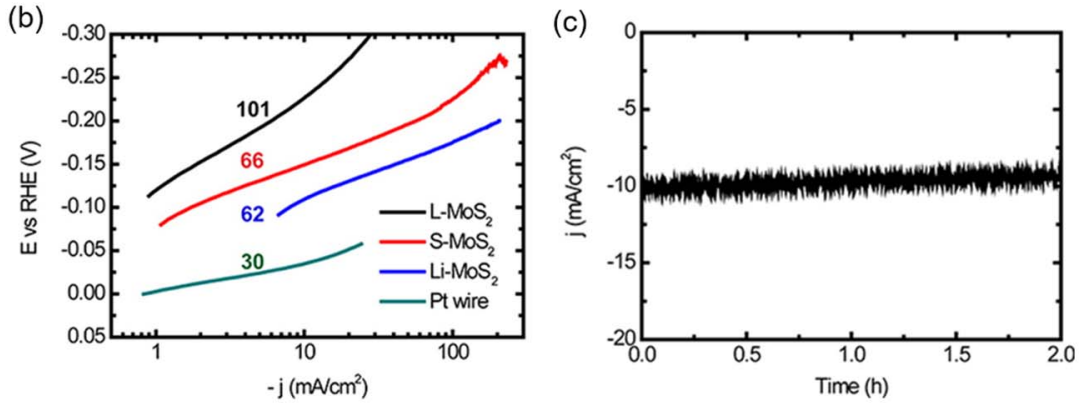

(f) ${ }^{0.0}-200 \mathrm{~mA} / \mathrm{cm}^{2}$

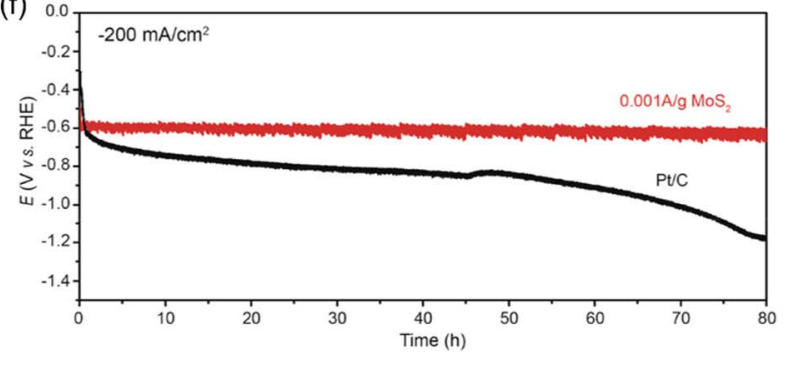

图 $6 \mathrm{Li}$ 插层 $\mathrm{MoS}_{2}$ 的催化活性研究 ${ }^{42,44}$

Fig. 6 Catalytic activity of $\mathrm{Li}$ intercalation $\mathrm{MoS}_{2}{ }^{42,44}$.

(a) Polarization curves of $\mathrm{L}-\mathrm{MoS}_{2}, \mathrm{~S}-\mathrm{MoS}_{2}, \mathrm{Li}-\mathrm{MoS}_{2}$, and Pt wire at a scan rate of $5 \mathrm{mV} \cdot \mathrm{s}^{-1}$. (b) Tafel plots of L-MoS $2, \mathrm{~S}-\mathrm{MoS}_{2}, \mathrm{Li}-\mathrm{MoS}_{2}$, and Pt wire. Li-MoS 2 shows the largest linear region. (c) Potentiostatic electrolysis of Li-MoS ${ }_{2}$ for $2 \mathrm{~h}$. The potential we applied is $118 \mathrm{mV} v s . \mathrm{RHE}$ after $i R$ correction. (d) LSV curves for the glass carbon electrode, commercial 20\% (w) Pt/C catalyst, and as-exfoliated $\mathrm{MoS}_{2}$ nanosheets (1.0-0.001 A·g-1). (e) Corresponding Tafel plots of the LSV curves in (d). (f) Chronopotentiometry curves of $0.001 \mathrm{~A} \cdot \mathrm{g}^{-1} \mathrm{MoS}_{2}$ QDs sheets and commercial $20 \%$ (w) Pt/C catalyst under a HER current density of $200 \mathrm{~mA} \cdot \mathrm{cm}^{-2}$ for $80 \mathrm{~h}$. Adapted from ACS Publications publisher. 
子、分子的数量有限, 可以减少电荷的注入量, 从 而避免相变的发生。例如THAB (四庚基溴化铵)等 分子的插入可以产生与金属离子插层类似的光电 性能增强等现象 ${ }^{40}$, 而由于其较大的体积和相对更 少的电荷注入, 不易在插层过程中发生相变或结 构扭曲, 显著提高了电化学过程的稳定性, 说明选 用合适尺寸的插层剂对插层法调整材料的电子性 能的重要性。

\section{2 光电性质的调控}

除了插层相变, 间隙内的客体与母体发生的相 互作用同样也会引起载流子浓度、光学透过率、电 导率和带隙特征的变化。例如嵌入石墨烯的铯原 子会极大程度的提高材料的费米能级，抑制带间 光学跃迁, 从而增加其在可见光范围内的透光率, 得到的石墨的光学性能显著改变 ${ }^{36}$ 。 $\mathrm{MoO}_{3}$ 中 $\mathrm{Sn}$ 的 插层也被证明大大提高了体系的电化学性能, 从 而具有更好的循环性能 ${ }^{37}$ 。过渡金属元素 $(\mathrm{Cu}$ 和 $\mathrm{Ge})$
也被报道插入到 $\mathrm{Si}_{2} \mathrm{Te}_{3}$ 的范德华间隙中, 电子带 隙、价带间过渡区、等离子体性质和潜在电导率都 发生了变化 ${ }^{38}$ 。在过渡金属插层过程中, 金属原子 可以改变材料的半导体行为, 增加带间的能量状 态, 或者改变材料的导电性, 同时相比于碱金属及 碱土金属, 过渡金属原子给电子的能力较弱, 插层 后并不倾向于与宿主本身紧密结合, 不会造成宿 主材料的整体结构改变。利用间隙中客体与宿主 材料的相互作用, 选择合适的插层剂, 是一种改善 材料光电特性的极有前景的手段。

2018年, Gong等人报道了一种基于溶剂的插 层方法可以实现 $\mathrm{Cu}$ 原子和 $\mathrm{Co}$ 原子在 $\mathrm{SnS}_{2}$ 中的可控 插入(如图7a) ${ }^{39}$ 。Gong等人发现, CVD生长的原始 $\mathrm{SnS}_{2}$ 是 $n$ 型半导体, 而嵌入铜和钴原子可以分别将 其调控为 $p$ 型半导体和高导电的金属态。生长的 $\mathrm{SnS}_{2} 、 \mathrm{Cu}-\mathrm{SnS}_{2}$ 和 $\mathrm{Co}-\mathrm{SnS}_{2}$ 的光学图像分别见图 7b, $c, d$ 。图7e为 $\mathrm{SnS}_{2} 、 \mathrm{Cu}-\mathrm{SnS}_{2}$ 和 $\mathrm{Co}-\mathrm{SnS}_{2}$ 的拉曼光谱。 (a)

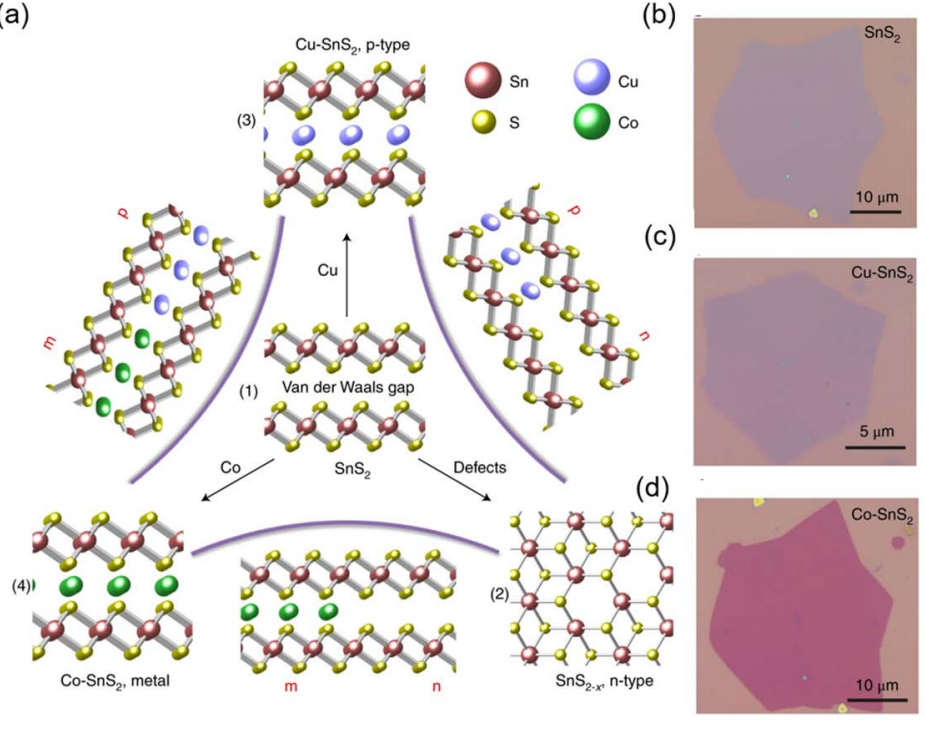

(e)

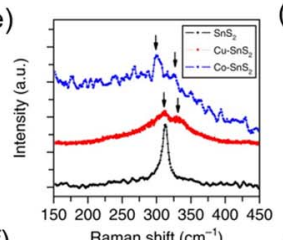

(f)

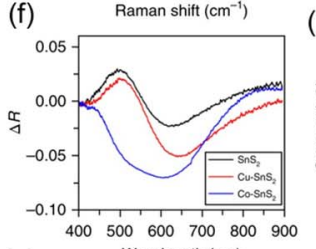

(g)

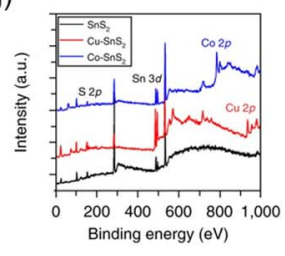

(h)
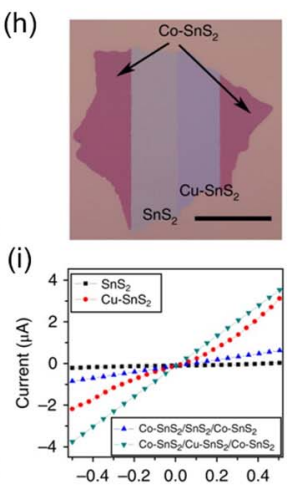

(j)

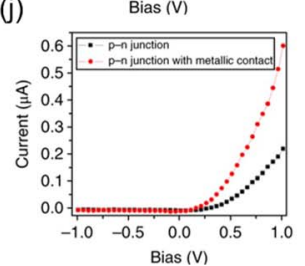

图 $7 \mathrm{Cu} 、 \mathrm{Co}$ 插层调控 $\mathrm{SnS}_{2}$ 光电性质的研究 ${ }^{39}$

Fig. 7 Study of inserting $\mathrm{Cu}$ or $\mathrm{Co}$ atoms to change the photoelectric properties of $\mathrm{SnS}_{2}{ }^{39}$.

(a) Schematics showing: (1) Bilayer pristine $\mathrm{SnS}_{2}$ with a van der Waals gap. (2) The S vacancy is the dominated defect type in the naturally CVD-grown SnS leading to an $n$-type semiconductor. (3) Cu-intercalated $\mathrm{SnS}_{2}$ as a $p$-type semiconductor. (4) Co-intercalated $\mathrm{SnS}_{2}$ as a highly conductive metal. Schematics between (2), (3) and (4) show that spatially controlled intercalation could realize the integration of these three elements. (b-d) Optical images of CVD-grown $\mathrm{SnS}_{2}, \mathrm{Cu}-\mathrm{SnS}_{2}$ and $\mathrm{Co}-\mathrm{SnS}_{2}$, respectively. Optical images clearly show that while the morphologies stay the same after intercalation, the colours of Cu-SnS 2 and $\mathrm{Co}-\mathrm{SnS}_{2}$ become more opaque and turn dark blue and violet-red, respectively, from the light blue colour of $\mathrm{SnS}_{2}$. (e) $\mathrm{Raman} \mathrm{spectra}$ of $\mathrm{SnS} \mathrm{S}_{2}$, Cu-SnS $\mathrm{S}_{2}$ and Co$\mathrm{SnS}_{2}$ (from trilayer samples). (f) Relative reflectance $\Delta R$ of $\mathrm{SnS}_{2}, \mathrm{Cu}-\mathrm{SnS}_{2}$ and Co-SnS $\mathrm{S}_{2}$ on a $\mathrm{SiO}_{2} / \mathrm{Si}$ substrate showing apparent spectral changes as a result of intercalation, indicating significantly different optical properties. $\Delta R$ is defined as $R\left(\right.$ flake $\left./ \mathrm{SiO}_{2} / \mathrm{Si}\right)-R(\mathrm{SiO} / \mathrm{Si})$. (g) Full XPS spectra show the presence of copper and cobalt after corresponding intercalation. (h) Demonstration of the ability to seamlessly integrate $n$-type $\mathrm{SnS}_{2}, p$-type $\mathrm{Cu}$-SnS $\mathrm{S}_{2}$ and $\mathrm{metallic}_{\mathrm{Co}-\mathrm{SnS}}$ within a single piece of nanosheet. Scale bars in G, $20 \mu \mathrm{m}$. (i) $I-V$ curves showing the nonlinear characteristics of $\mathrm{SnS}_{2}$ or $\mathrm{Cu}-\mathrm{SnS} \mathrm{S}_{2}$ with a Ti/Au electrode, and $\mathrm{Co}-\mathrm{SnS}_{2}$ can be used as the ohmic contact for both $\mathrm{SnS}_{2}$ and $\mathrm{Cu}-\mathrm{SnS}_{2}$. (j) Typical rectification behaviour of the $\mathrm{SnS}_{2} / \mathrm{Cu}_{-} \mathrm{SnS}_{2}$ heterojunction, whose performance can be further improved by using $\mathrm{Co}-\mathrm{SnS}_{2}$ as contact. Adapted from Springer Nature publisher. 
插层前后样品在二氧化硅/硅祄底上也表现出改变 的相对反射率, 表明 $\mathrm{Cu}$ 和 $\mathrm{Co}$ 的成功插入(如图7f), XPS光谱进一步证实插层后铜和钴的存在 (如图 $7 \mathrm{~g}$ )。原始 $\mathrm{SnS}_{2}$ 晶格在与层间 $\mathrm{Cu}$ 原子的作用下, $\mathrm{Cu}$ 原子中扩展的 $s$ 电子可以部分填充硫化锡的导带, 同 时由于客体与宿主晶格原子的耦合作用, $\mathrm{C}_{1}$ 和 $\mathrm{C}_{2}$ 带的能量差距越来越小, 从而使 $\mathrm{Cu}-\mathrm{SnS}_{2}$ 表现出具 有窄带隙的 $p$ 型半导体特征。Co原子未填充的 $d$ 电 子与原始二硫化锡的能带发生耦合, 造成费米能 级处杂化态部分被填满, 从而表现出显著提高的 导电性。相比于 $\mathrm{CVD}$ 生长的原始 $n$ 型 $\mathrm{S}$ 空位 $\mathrm{SnS}_{2}, \mathrm{Cu}$ 掺杂的双层 $\mathrm{SnS}_{2}$ 的场效应迁移率能够达到 40 $\mathrm{cm}^{2} \cdot \mathrm{V}^{-1} \cdot \mathrm{s}^{-1}$, Co 掺杂的 $\mathrm{SnS}_{2}$ 则表现出高导电的金属 性能。这种插层技术同时实现了在原子级尺度二 维半导体材料的 $p$ 型、 $n$ 型及非简并型掺杂。Gong 等人还进一步利用光刻技术第一次实现了 $n 、 p$ 型 半导体和金属二维材料的无缝连接和集成, 实现 了精确的尺寸和空间控制(见图7h), 并进行了一系 列性能测试(如图7i, j)。2018年, $\mathrm{Li}$ 等人报道了在 二维层状石墨化氮化碳 $\left(\mathrm{g}-\mathrm{C}_{3} \mathrm{~N}_{4}\right)$ 中利用氧在层内 修饰并同时在层间插入钾的新结构 46 。氧的掺杂和 层间插入的钾形成一种内部范德华异质结构, 氧 修饰的 $\mathrm{CN}$ 层与相邻的 $\mathrm{CN}$ 子层之间建立了一个范 德华异质结, 同时, $\mathrm{K}$ 的插入可以加速层间电荷流, 作为中介加强了层间的范德华相互作用。合成的 O、 $\mathrm{K}$ 功能化 $\mathrm{g}-\mathrm{C}_{3} \mathrm{~N}_{4}$ 具有显著增强的光催化活性和 选择性, 与原始 $\mathrm{g}-\mathrm{C}_{3} \mathrm{~N}_{4}$ 相比, 净化效率几乎提高了 $100 \%$ 。

\section{3 量子性质的改变}

客体与宿主材料的相互作用也会引起一些其 他性质, 如磁性、拓扑磁相和超导特性的变化。2016 年, Farrell等人报道了将 $\mathrm{Au}$ 嵌入石墨烯和六方氮 化硼的范德瓦尔斯异质结构中可以诱导出石墨烯 和金原子之间的强杂化效应, 从而产生约 $25 \mathrm{meV}$ 的Rashba相互作用(自旋轨道相互作用)。金的负磁 电阻高达 $75 \%$, 表明层间的金形成了磁矩, 反常霍 尔效应表明可能形成了有序磁相, 这些作用可能 打开了通向拓扑磁态的大门 ${ }^{47}$ 。

2017年, Zhao等人报道了一组新的层状硫系 化合物 ${ }^{48} 。 \mathrm{~A}_{x} \mathrm{Cd}_{x} \mathrm{Bi}_{4-x} \mathrm{Q}_{6}(\mathrm{~A}=\mathrm{Cs}, \mathrm{Rb}, \mathrm{K} ; \mathrm{Q}=\mathrm{S}$ 和 $\mathrm{A}=\mathrm{Cs} ; \mathrm{Q}=\mathrm{Se}$ )在密度泛函理论 (DFT)水平上的能 带结构计算表明, $\mathrm{Cs}_{1.13} \mathrm{Cd}_{1.13} \mathrm{Bi}_{2.87} \mathrm{Se}_{6}$ 和 $\mathrm{Cs}_{1.43} \mathrm{Cd}_{1.43} \mathrm{Bi}_{2.57} \mathrm{~S}_{6}$ 是直接带隙半导体。多晶 $\mathrm{Cs}_{1.43} \mathrm{Cd}_{1.43} \mathrm{Bi}_{2.57} \mathrm{~S}_{6}$ 样品呈 $n$ 型传导, 在 $773 \mathrm{~K}$ 处导热 系数极低。 $\mathrm{Cs}_{1.43} \mathrm{Cd}_{1.43} \mathrm{Bi}_{2.57} \mathrm{~S}_{6}$ 层间的铯离子是可移 动的, 可以在水溶液中与 $\mathrm{Pb}^{2+} 、 \mathrm{Zn}^{2+} 、 \mathrm{Co}^{2+} 、 \mathrm{Cd}^{2+}$
拓扑交换。这些性质的显著改变表明了范德华间 隙中金属阳离子的嵌入是改变材料的热学性能和 能带结构的一种有效软化学途径。

2019年, Wang等人报道了利用电化学插层的 有机分子形成杂化超晶格大大提高了 $\mathrm{Cr}_{2} \mathrm{Ge}_{2} \mathrm{Te}_{6}$ 的 铁磁转变温度 ${ }^{41}$ 。Wang 等人通过四丁基铵 $\left(\mathrm{TBA}^{+}\right)$ 的电化学插层形成杂化超晶格( $\mathrm{TBA}) \mathrm{Cr}_{2} \mathrm{Ge}_{2} \mathrm{Te}_{6}$, 插 层前后的晶体结构如图 $8 \mathrm{a}, \mathrm{b}$, XRD图谱(如图8c) 可以看出插层衍射峰整体向小角方向移动。研究 发现(TBA) $\mathrm{Cr}_{2} \mathrm{Ge}_{2} \mathrm{Te}_{6}$ 表现出金属行为, 并且居里温 度从原始 $\mathrm{Cr}_{2} \mathrm{Ge}_{2} \mathrm{Te}_{6}$ 半导体的 $67 \mathrm{~K}$ 显著提高到 208 $\mathrm{K}$ (如图8d), 磁易轴从 $c$ 轴重定向到 $a b$ 平面(如图 $8 \mathrm{~d}-\mathrm{g})$ 。理论计算表明, $\mathrm{Cr}_{2} \mathrm{Ge}_{2} \mathrm{Te}_{6}$ 层被电子掺杂, 基态由半导体转变为金属。因此, 铁磁性来自于 (TBA) $\mathrm{Cr}_{2} \mathrm{Ge}_{2} \mathrm{Te}_{6}$ 中传导电子与局部自旋的耦合, 而 不是来自于 $\mathrm{Cr}_{2} \mathrm{Ge}_{2} \mathrm{Te}_{6}$ 中微弱的超交换作用。在费 米能级附近, 态密度主要贡献来自先前未被占用 的 $\mathrm{Cr}$ 元素的 $d_{x z}$ 和 $d_{y z}$ 轨道, 这些导电的 $d_{x z}$ 和 $d_{y z}$ 轨道 将与 $\mathrm{Cr}$ 的其他局部自旋轨道产生Hund耦合交换, 满足Anderson和Hasegawa提出的双交换铁磁性相 互作用机理。由于直接耦合, $\mathrm{Cr}$ 自旋之间的磁耦合 强度显著增加, 这使得居里温度大幅提高, 同时 $\mathrm{Cr}$ 离子的自旋轨道耦合以及双重交换相互作用使易 磁轴从 $\mathrm{Cr}_{2} \mathrm{Ge}_{2} \mathrm{Te}_{6}$ 的 $c$ 轴方向向 (TBA) $\mathrm{Cr}_{2} \mathrm{Ge}_{2} \mathrm{Te}_{6}$ 的 $a b$ 面变化。该研究表明有机分子的电化学嵌入可 以显著地调整范德华材料的磁性, 这为操纵磁性 范德华晶体的磁性和电子性质开辟了一条新途 径。

在上面的讨论中, 在二维材料的范德华间隙 引入的客体可以与原有晶格原子发生多种相互作 用, 在电、光、热、磁等多个方面调控材料的性质。 这些工作说明, 利用范德华间隙中的客体与母体 的相互作用可以创造综合性能更加优异的二维材 料, 作为改变二维材料性能的一种通用方法有极 大的潜力, 在二维材料的性能调控方向上有重要 意义。

\section{4 客体原子带来新性质}

插入二维材料范德华间隙的客体原子不仅可 以与宿主材料相互作用改变其电、光性质, 还可以 带来一些宿主材料原本所并不具备的性质, 如优 异的催化性质、铁磁和超导性质。

\section{1 催化}

在催化领域, 限域生长这一新兴的概念是设 计和合成新型二维纳米结构材料的一个很有前景 的策略。范德华间隙可提供催化剂限域生长的空 
(a)

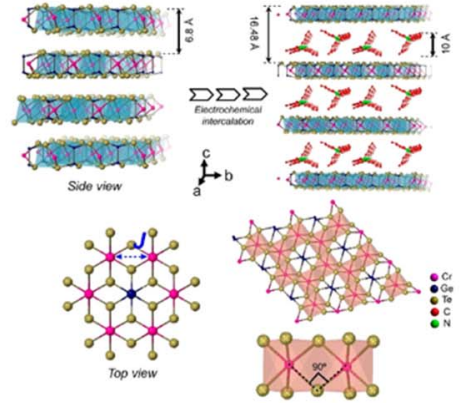

(c)

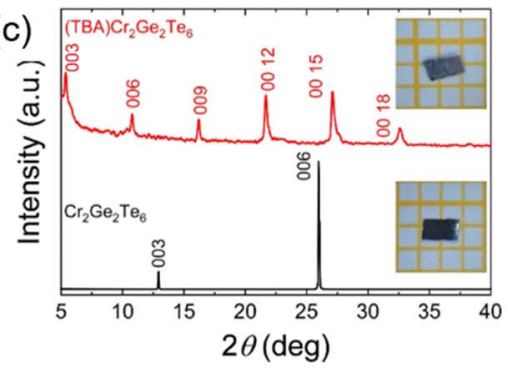

(d)

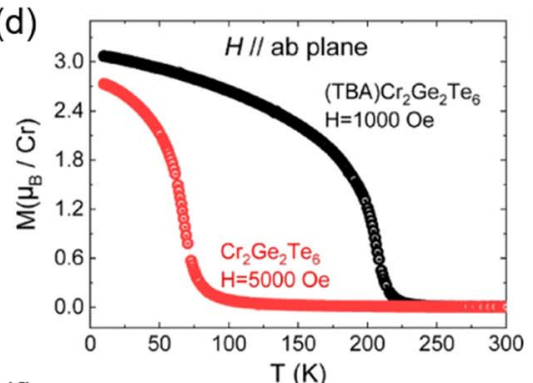

(f)

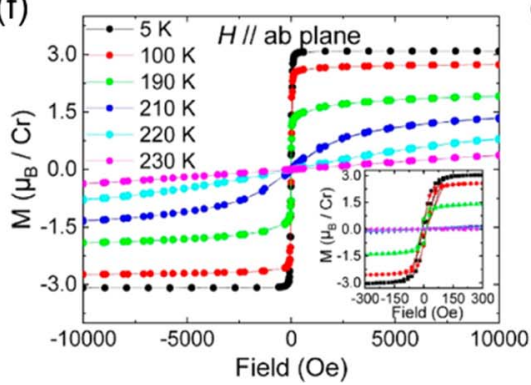

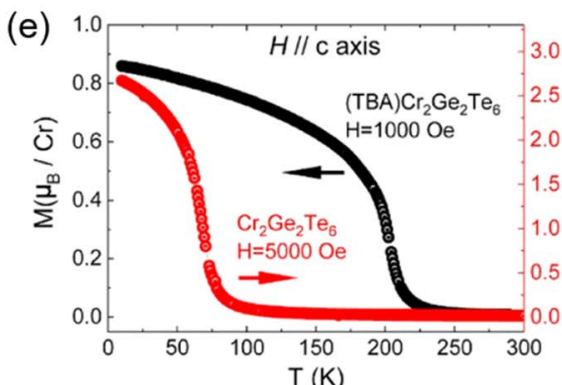

(g)

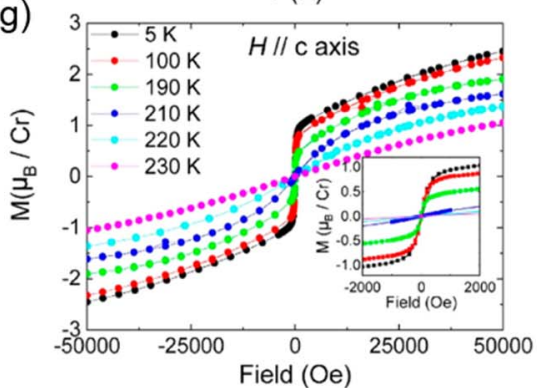

图 8 合成了有金属性的( $\mathrm{TBA}) \mathrm{Cr}_{2} \mathrm{Ge}_{2} \mathrm{Te}_{6}$ 杂化超晶格, 提高其铁磁转变温度 ${ }^{41}$

Fig. 8 Synthesized a (TBA) $\mathrm{Cr}_{2} \mathrm{Ge}_{2} \mathrm{Te}_{6}$ hybrid superlattice with metallic behavior, and the Curie temperature is significantly increased ${ }^{41}$.

(a) Side-view crystal structure of $\mathrm{Cr}_{2} \mathrm{Ge}_{2} \mathrm{Te}_{6}$ and (TBA) $\mathrm{Cr}_{2} \mathrm{Ge}_{2} \mathrm{Te}_{6}$. (b) Left: Top-view structure of $\mathrm{Cr}_{2} \mathrm{Ge}_{2} \mathrm{Te}_{6}$. $\mathrm{Right}_{\text {: Edge-sharing CrTe }}$ octahedrons form a honeycomb-like structure in the ab-plane and $\mathrm{Cr}$ spins interact through indirect $90^{\circ}$ superexchange couplings with $\mathrm{Te}$. (c) $\mathrm{XRD}$ patterns of pristine $\mathrm{Cr}_{2} \mathrm{Ge}_{2} \mathrm{Te}_{6}$ and intercalated (TBA) $\mathrm{Cr}_{2} \mathrm{Ge}_{2} \mathrm{Te}_{6}$ showing a series of (001) diffractions. (d, e) Temperature-dependent magnetic susceptibility $(M-T)$ of pristine $\mathrm{Cr}_{2} \mathrm{Ge}_{2} \mathrm{Te}_{6}$ and (TBA) $\mathrm{Cr}_{2} \mathrm{Ge}_{2} \mathrm{Te}_{6}$ with $H / / a b$ and $H / / c$, respectively. (f, g) Magnetic field dependent magnetic susceptibility $(M-H)$ of $\left(\mathrm{TBA} \mathrm{Cr}_{2} \mathrm{Ge}_{2} \mathrm{Te}_{6}\right.$ with $H / / a b$ and $H / / c$,

respectively. Adapted from ACS Publications publisher.

间。在二维材料的层间引入高度分散的纳米粒子 和团簇近年来也被发现是实现创造高度优异性能 催化剂的有效方法, 所得到材料的优异的催化特 性往往是原有的材料所不具备的。范德华间隙中 的纳米粒子在层间的限域作用下能够保持很好的 分散性, 从而提高了在催化过程中的稳定性, 为高 性能稳定催化奠定了基础, 而如何将粒子引入二 维材料层间构建高效的催化体系具有一定的挑战 性。Ebina在一篇多相催化的工作中报道了将 $\mathrm{RuO}_{2}$ 引入 $\mathrm{KCa}_{2} \mathrm{Nb}_{3} \mathrm{O}_{10}$ 中, 但并未详细研究层间存在的 $\mathrm{RuO}_{2}$ 与宿主材料的相互作用 ${ }^{49}$ 。Mallouk等人报道 了成功通过预插层的方法将金纳米颗粒引入到层 状固体中 50,51 。但是, 该体系存在一定的局限性, 如层间空间残留的元素难以完全去除, 还存在层 间的金与固体表面之间存在直接结合的问题。 Mallouk及其同事还报告了另一种系统, 利用 $\mathrm{Rh}-$ $\mathrm{O}-\mathrm{Nb}$ 键的共价相互作用, 成功将高度分散的 $\mathrm{Rh}(\mathrm{OH})_{3}$ 纳米颗粒插入 $\mathrm{KCa}_{2} \mathrm{Nb}_{3} \mathrm{O}_{10}$ 的层间, 表现出 了比铂插层更高的光催化活性 ${ }^{52}$, 但反应过程中整 体的水裂解并不成功, 也没有讨论方法对其他金 属的适用性。Oshima等人报道了利用金属配合物
和带负电的二维 $\mathrm{Ca}_{2} \mathrm{Nb}_{3} \mathrm{O}_{10}$ 薄片之间的静电作用 在其层间空间沉积了直径小于 $1 \mathrm{~nm}$ 的金属团簇(包 括铂等团簇), 且沉积过程不需要额外试剂。在一 定条件下, 该材料表现出优异的光催化特性, 可以 将水分解为氢气和氧气 ${ }^{53}$ 。透射电镜表示Pt的分散 性很高, 沉积的铂纳米粒子大多直径小于 $1 \mathrm{~nm}$ 。当 一些铂聚集形成较大的二次粒子时, 其直径仍然 小于 $4 \mathrm{~nm}$ 。此外, 插入Pt纳米团簇与纳米片表面发 生相互作用, XPS显示半导体与助催化剂之间的 密切接触也能有效提高光催化反应的效率。在该 工作中, Oshima等人还进一步在纳米层间空间引 入了 $\mathrm{Pd} 、 \mathrm{Rh}$ 和 $\mathrm{Ru}$ 等纳米颗粒并进行相关研究, 证 明了该插层方法的普适性。2018年，Luo等人报道 了一种二维 $\mathrm{MoS}_{2}$ 限域的 $\mathrm{Co}(\mathrm{OH})_{2}$ 纳米颗粒电催化 剂的设计和合成, 该催化剂可以加速水的分解, 并 在碱性溶液中表现出良好的耐久性, 从而显著提 高了催化性能 ${ }^{5}$ 。 $\mathrm{Co}(\mathrm{OH})_{2}$ 纳米颗粒加速了水的解 离, 同时 $\mathrm{MoS}_{2}$ 纳米片加速了氢气的生成。电化学 测试表明该纳米片在碱性条件下优越的催化活性 和出色的稳定性。此外密度泛函理论计算表明 $\mathrm{Co}(\mathrm{OH})_{2}$ 纳米粒子和 $\mathrm{MoS}_{2}$ 纳米片的共同作用促进 
了催化活性提高, 限域作用很好的约束了层间的 纳米粒子, 保证了催化过程的稳定。原则上, 这一 策略可以推广到其他宿主材料(如石墨烯、氙、黑 磷和过渡金属二卤化物) 和插层剂 (如 $\mathrm{Ni}(\mathrm{OH})_{2}$ 等), 为其他电化学反应提供有效的催化剂。

除了在层间插入纳米颗粒, 二维范德华间隙的 单原子插层催化剂最近被报道具有广阔的应用前 景。Chen等人报道了一种具有垂直结构的钴单原 子嵌入 $\mathrm{MoS}_{2}$ 的催化剂(如图9a-c), 由于间隙空间 的限域效应和与基底较强的耦合作用, 体系的电 子结构得到改变, 进而提出了自由基介导硫化物 氧化的途径(如图9d) ${ }^{55}$ 。该单原子催化剂表现出较 强的吸附和边缘选择性氧化作用, 可以实现硫醚
的选择性氧化。一系列理论计算研究了催化剂中 的电荷密度分布和对不同物种的吸附能, 并进行 $E P R$ 实验研究了该催化剂的催化反应过程(见图 $9 \mathrm{e}-\mathrm{g}$ )。该催化剂可以催化合成一系列复杂的砜和 亚砜类中间体和药物衍生物, 在34种有机分子的 合成中选择性高达 $99 \%$, 具有非常重要的工业应用 价值。

如上文所提到的，二维材料中金属原子的插 层已受到研究工作者的广泛关注。这种方法可以 运用在不同种的二维材料体系中, 并插入不同种 类的客体金属原子。由于范德华间隙的限域效应, 层间的金属原子可以被稳定, 维持其原子级的分 立, 构建具有相当优异催化性能的单原子催化剂。

(a)
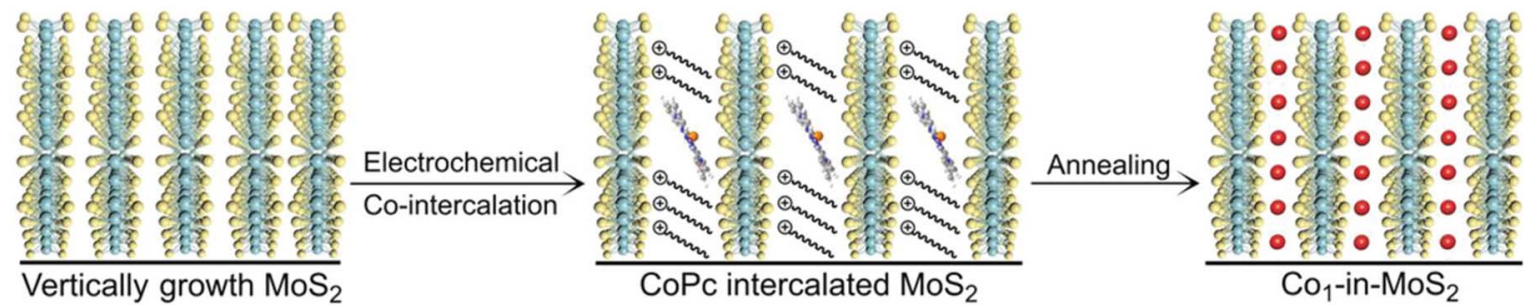

gos $\mathrm{MO}_{2}$.

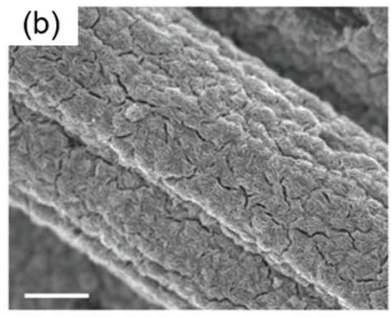

(e)

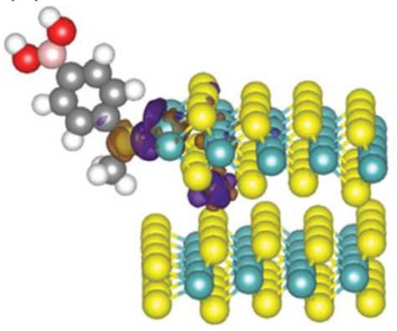

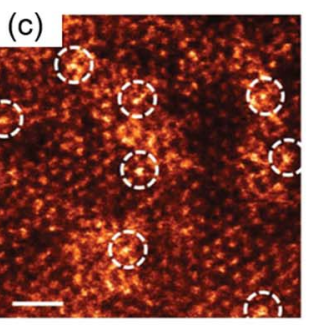

(f)

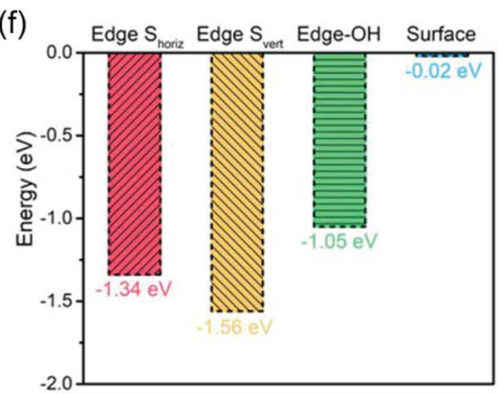

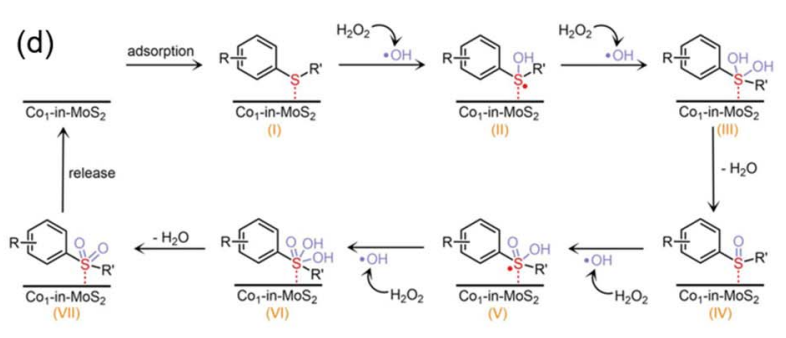

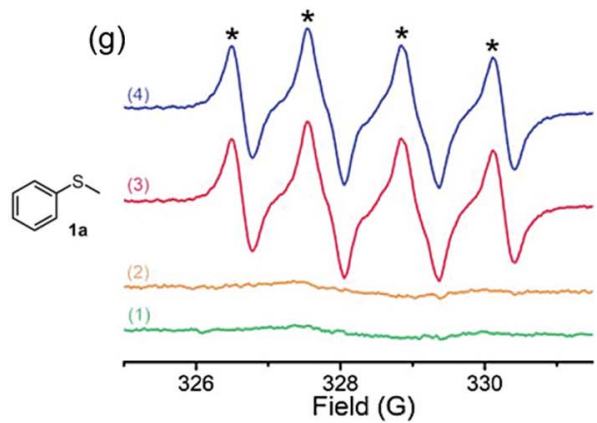

图 9 钴单原子嵌入 $M_{0} S_{2}$ 的催化剂合成及催化机理 55

Fig. 9 Synthesis and catalytic mechanism of cobalt single atom-intercalated $\mathrm{MoS}_{2}{ }^{55}$.

(a) Schematic of the electrochemical co-intercalation and subsequent annealing process to convert the intercalants into single atom catalyst. (b) SEM image showing the vertical structure of $\mathrm{MoS}_{2}$ array. (c) Atomic-resolution STEM-HAADF image of $\mathrm{Co}_{1}$-in-MoS 2 . Scale bar: (b) $2 \mu$ m; (c) $1 \mathrm{~nm}$. (d) Proposed radical mediated pathway for sulfide oxidation. (e) Differential charge density of the optimized adsorption configuration (side view) of the sulfide 1q at the edge of bilayered $\mathrm{Co} 1-\mathrm{in}-\mathrm{MoS}_{2}$. Purple and brown region represent the isosurface of the electron lost and gained. The color scheme used: dark cyan for Mo, yellow for S, blue for Co, gray for C, red for O, pink for B and white for H. (f) Difference in adsorption energies for various adsorption configurations of sulfide 1q on $\mathrm{Co}_{1}$-in- $\mathrm{MoS}_{2}$. (g) EPR experiments on trapping DMPO-OH adduct (marked as *) in sulfide oxidation. (1) Without $\mathrm{H}_{2} \mathrm{O}_{2}$ and catalyst; (2) without $\mathrm{H}_{2} \mathrm{O}_{2}$; (3) without sulfide 1a; and (4) standard conditions. Adapted from Wiley publisher. 
然而, 目前关于此类单原子催化剂的研究还较少。 我们在此提出以下一些设想:

(1)在单原子催化十分热门的今天, 我们能否 拓展一种新的且较为普适的通过插层二维材料获 得一系列高稳定性的特殊单原子催化剂的方法? 相对于面内掺杂获得的单原子催化剂, 层间插层 的客体单原子并不一定会与宿主晶格原子发生共 价成键, 耦合形式的不同可能也意味着这种方式 制备出的单原子催化剂可能具有不同的催化反应 途径, 从而展现出不同的反应机理和催化活性。

(2)对于本身就具有优异催化性质的贵金属, 将其作为单原子分散在二维材料的层间能否进一 步显著提升其催化性能? 现有的一些贵金属催化 剂通常存在催化稳定性较差和成本过高等问题, 极 大的限制了这些体系的真正应用。层间限域构建 这些贵金属的相应的单原子催化剂也许是提高其 催化稳定性和利用效率, 从而提高其实际应用价 值的潜在方法。现有的层间插入的单原子催化剂 研究仍比较有限, 对于贵金属原子插层后与层状 主体材料之间的耦合也还需进一步的探究。

(3)对于本身催化性质较差的金属元素, 将其 作为单原子分散在二维材料层间能否获得优异的 催化性能? 不同金属元素插层过程都会引起能带 结构甚至晶体结构的改变, 并可能会引起宿主材 料催化活性及催化位点的改变。我们认为通过对

(a)

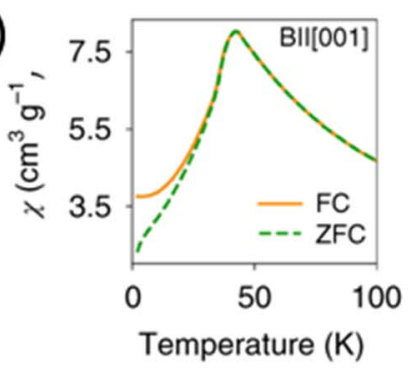

(b)

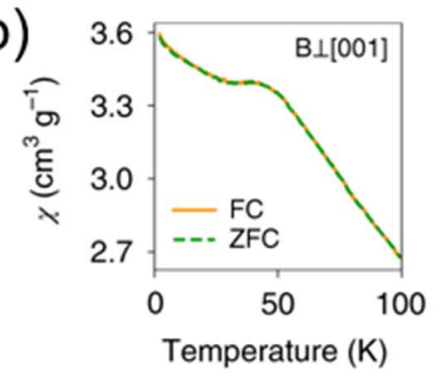

电子和能带结构进行合理设计, 选择合适的金属 元素和宿主材料, 或许可以构建出非贵金属的层 间限域单原子催化剂, 促进低成本高产量催化剂 和催化体系的进一步开发研究。

\section{2 磁性}

除了上文所提到优异的催化性能, 二维范德 华间隙的客体原子也会带来原来材料并不具备的 磁性。2019年, Nair等人报道了磁性插层过渡金属 二卤化物 $\mathrm{Fe}_{1 / 3} \mathrm{NbS}_{2}$ 在 $42 \mathrm{~K}$ 下表现出反铁磁有序 性, 铁原子被插在 $\mathrm{NbS}_{2}$ 的 $2 \mathrm{H}$ 相平面间的周期晶格 中 56 。他们发现该材料在较低的 $10 \mathrm{~A} \cdot \mathrm{cm}^{-2}$ 数量级的 电流密度下会发生磁序的重新定向, 反向自旋电 效应和插层后破碎的晶格镜面对称导致了出现反 铁磁特性(如图10)。这一发现表明插层 $\mathrm{Fe}$ 等客体原 子的 TMDs 在创建新型铁磁或反铁磁材料中的应 用潜力。

2020年, Zhao等人也报道了 Ta插入的自插层 $\mathrm{Ta}_{7} \mathrm{~S}_{12}$ ic-2D晶体中出现了铁磁性 ${ }^{57}$ 。 $\mathrm{Ta}_{7} \mathrm{~S}_{12}$ 插入 $\mathrm{Ta}$ 的原子结构模型如图 $11 \mathrm{a}-\mathrm{c}$, 生长模式如图 $11 \mathrm{~d}$ 磁输 运测量表明 $30 \mathrm{~K}$ 以下观察到温度依赖性电阻率非 饱和上升, 在低温下可以观察到高达 $9 \mathrm{~T}$ 的线性磁 电阻(见图11e-g)。此外在该体系中观察到了反常 霍尔效应。Zhao等人针对该体系进行了一系列的 理论研究, DFT计算表明完美的双分子层 $2 \mathrm{H}_{\mathrm{a}}$-堆叠 $\mathrm{TaS}_{2}$ 具有非磁性基态, 而插入的 $\mathrm{Ta}$ 与原始晶格发

图 10 插层过渡金属二卤化合物 $\mathrm{Fe}_{1 / 3} \mathrm{NbS}_{2}$ 的结构和磁性测试 56

\section{Fig. 10 Structure and magnetic measurements on the intercalated transition metal dichalcogenide $\mathrm{Fe}_{1 / 3} \mathrm{NbS}_{2}{ }^{56}$.}

(a) Magnetization $(\chi)$ measurements with field along the [001] direction ( $c$ axis) show a peak at $42 \mathrm{~K}$ in both the field cooled and zero-field cooled traces indicating the presence of an AFM transition. (b) In-plane magnetization measurements also show a weak peak at $42 \mathrm{~K}$. A cooling field of $0.1 \mathrm{~T}$ was used for both a and b. (c) The crystal structure of $\mathrm{Fe}_{1 / 3} \mathrm{NbS}_{2}$ is that of $2 \mathrm{H}-\mathrm{NbS}_{2}$ with iron atoms intercalated between layers. Adapted from Springer Nature publisher. 
(a)

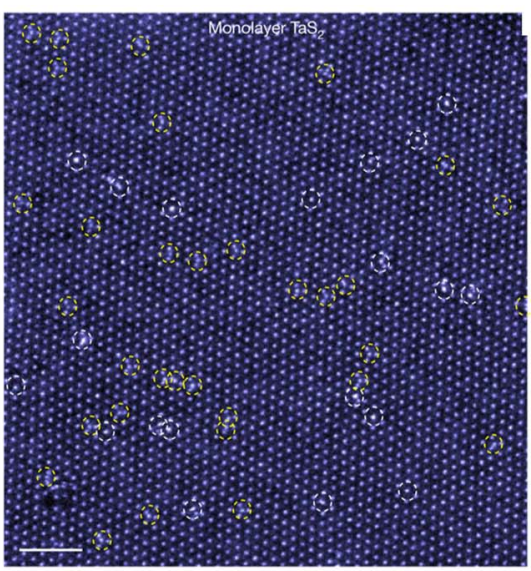

(h)

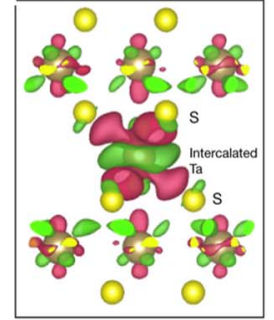

(k)

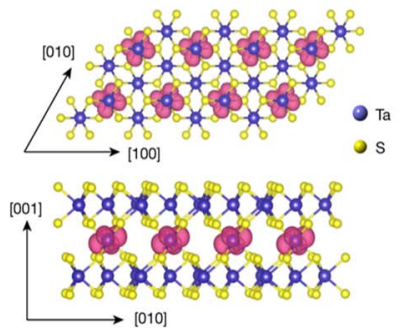

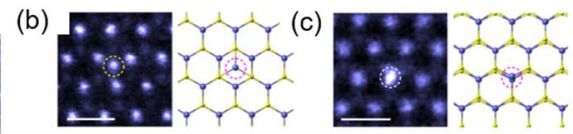

(d)

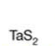

Growth mechanism

(e)

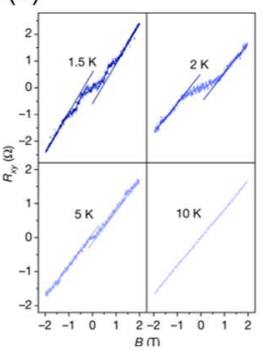

$\mathrm{Ta}-\mathrm{TaS}_{2}$

$\mathrm{TaS}_{2}-\mathrm{Ta}^{-} \mathrm{TaS}_{2}$

senson

Soratom

(f)

(g)
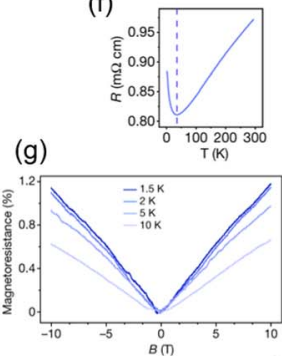

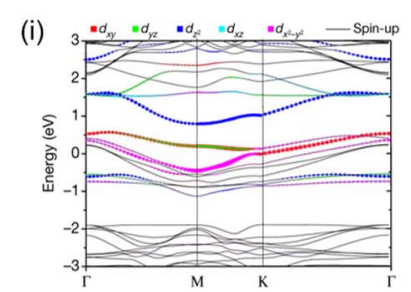

(I)
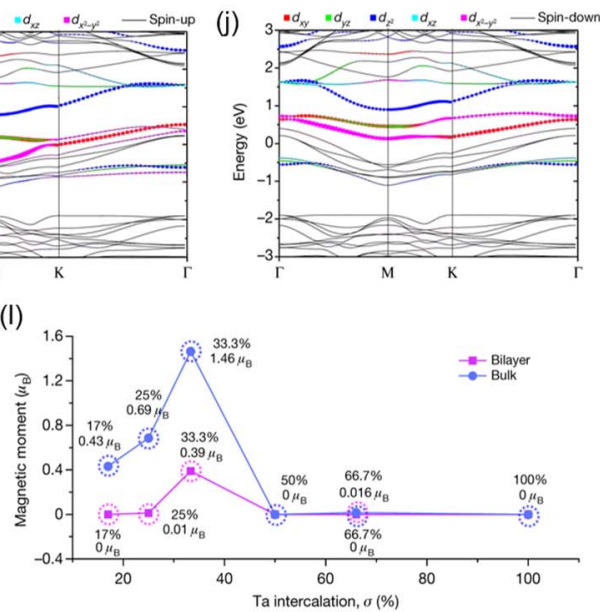

图 $11 \mathrm{Ta}$ 插入的自插层 $\mathrm{Ta}_{7} \mathrm{~S}_{12}$ ic-2D 晶体研究 ${ }^{57}$

Fig. 11 Studies of self-intercalated $\mathrm{Ta}_{7} \mathrm{~S}_{12}$ crystal ${ }^{57}$.

(a) Showing an abundance of interstitial Ta atoms at the center of honeycomb (b) or on top of the Ta site (c). (b, c) The corresponding atomic models are depicted on the right. (d) Schematic depicting the layer-by-layer growth of ic-2D crystals. (e, g) Hall resistance $\left(R_{x y}\right)(\mathrm{e})$ and Temperature-dependent magnetoresistance ( $\mathrm{g}$ ) of $\mathrm{Ta}_{7} \mathrm{~S}_{12}$ under an out-of-plane magnetic field. (f) Resistivity of the $\mathrm{Ta}_{7} \mathrm{~S}_{12}$ ic-2D crystal as a function of temperature. (h) Contour plot of charge density difference in Ta-intercalated $\mathrm{Ta}_{7} \mathrm{~S}_{12}$. (i, j) Orbital-resolved spin-up (i) and spin-down (j) band structures of the intercalated Ta in $\mathrm{Ta}_{7} \mathrm{~S}_{12}$. (k) Top view (top) and side view (bottom) spin density isosurface of Ta-intercalated $\mathrm{Ta}_{7} \mathrm{~S}_{12}$. (1) Calculated magnetic moments as a function of the Ta-intercalation concentration $(\sigma)$ in $2 \mathrm{H}_{\mathrm{a}}$-stacked nonstoichiometric $\mathrm{Ta}_{x} \mathrm{~S}_{y} \cdot \mu_{\mathrm{B}}$, Bohr magneton. Adapted from Springer Nature publisher.

生电子转移, 引入额外的穿过费米能级的自旋分 裂能带, 从而形成磁基态。磁矩分布在插层 $\mathrm{Ta}$ 原子 的 $d$ 轨道上, 费米能级由原始 $\mathrm{Ta}$ 轨道与插层 $\mathrm{Ta}$ 轨道 自旋带杂化而成。只有插入的 $\mathrm{Ta}$ 原子表现出净的 自旋密度(如图11h-1)。这些工作表明插层客体原 子到二维材料的范德华间隙不仅仅可以调控材料 原有的物理化学性质, 还可以产生许多新的性质。 这些发现对未来研究和丰富的二维材料性能, 进 一步扩大二维材料的应用领域具有极其重要的 意义。

\section{5 层间存储：锂离子和锂金属电池等}

层间存储是锂离子二次电池的核心。在层状
材料的层间可逆脱插金属离子可以实现可充电电 池的能量存储和释放。近年来, 锂离子电池、钠离 子电池、锂硫电池和其他类型的电池得到了大量 科研工作者的关注 ${ }^{58-63}$, 在能源存储领域展现出极 其出色的应用潜力。其中由于插层的难度较低, 对 锂离子电池的研究开始的最早, 而随着有机分子 插层柱化等方法的应用和各种二维体系的涌现, 层间间距进一步被扩大, 钠离子、镁离子、钾离子、 锌离子等电池体系也因此迅速发展起来。层间工 程和表面修饰方法开始被研究工作者广泛地应用 于提升锂离子电池的速率和容量。层间存储是能 源存储领域至关重要的一部分。

锂离子电池是正负电极之间传输的离子为 $\mathrm{Li}^{+}$ 
的电池体系, 关于锂基电池的研究最早可追溯到 上个世纪早期。1913年, Gilbert N. Lewis在美国化 学学会会刊上就发表了系统阐述和测量金属锂电 化学电位的工作, 被视为最早的系统研究锂金属 电池的工作 ${ }^{64}$ 。在随后的几十年里不少科研工作者 都尝试过锂金属电池的产业化。1965年, 德国化学 家Walter Rüdorff首次发现在一种层状结构的硫化 物 $\mathrm{TiS}_{2}$ 中可以化学嵌入锂离子 65 , 在 70 年代时任美 国埃克斯石油公司科学家Stanley Whittingham经 过一系列细致研究进一步证明了这种层状结构的 金属硫化物 $\left(\mathrm{TiS}_{2}\right)$ 可以在层间实现锂的电化学可逆 储存, 并以此为基础构建了一个锂金属二次可充电 池原型 66,67 。尽管可逆锂电在原理上成功得到印 证, 但由于金属锂作为负极在不断循环中容易生 成树枝状的锂枝晶从而造成电池内部短路引发起 火爆炸, 锂金属电池的枝晶问题成为阻碍其产业 化应用的巨大问题。虽然对于锂金属电池的应用 研究遇到困难, 科研工作者还是继续提出了构建 出可实现锂离子在充放电过程中来回穿梭的新电 池体系, 锂离子电池的概念开始酝酿。1980年, 牛 津大学无机化学系教授John B. Goodenough提出 用一种含锂的金属氧化物来做为锂电池正极, 并 最终使用了具有层状结构的钴酸锂 $\left(\mathrm{LiCoO}_{2}\right)$, 从而 为当时还并不清晰的锂离子电池体系维形寻找到 了理想的正极材料 68 。几乎在同一时期, 人们发现 可以采用石油焦来代替金属锂作为锂离子电池的 负极材料。虽然石油焦存在着比容量较低的问题, 后来先后被中间相碳微球和人造石墨负极或改性 天然石墨负极所取代, 但在当时钴酸锂正极和碳 负极的发现, 仍然为商用锂离子电池的诞生奠定 了基础。1991年, 首批商业化的锂离子电池在索尼 公司诞生, 由于其自身具有的优势(高输出电压、 高容量和稳定的嵌入式材料结构), 锂离子电池得 到了科研工作者们大量的关注和研究, 得到了蓬 勃的发展。

近年来, 二维材料的蓬勃发展进一步为锂离 子电池提供了可选择的电极材料, 此外, 层间工程 与具有更大层间距的材料体系也在不断被发现, 促 进了钠、钾离子和其他多价离子电池的发现和研 究。早期对锂离子电池的研究重点探讨了各种材 料体系中插层过程的反应机理和电极表面结构的 变化。插层过程往往会引起一定程度的晶格畸变, 而这一过程往往伴随着相变, 这是决定锂电池动 力学和可逆性的关键因素之一。2013年, Mashtalir 等人报道了联氨的插层及其与 $N, N$-二甲基甲酰胺 共插层导致表面功能化并进行超声处理得到的
MXenes (二维过渡金属碳化物、氮化物或碳氮化 物)纸的电池性能, 发现其在较高的充电率下表现 出优异的锂离子容量 69 。Eames等人进一步报道了 二维过渡金属碳化物MXenes对各种离子的插层 潜力, 并使用密度泛函理论进行了相关计算。插层 离子的大小决定了其进入MXenes层间的难易, 同 时表面官能团化和过渡金属的种类对 MXenes的 电压和容量有显著的影响。为探索高容量的 MXenes电池和其他离子的电池体系提供了有价 值的见解 70 。少层的 $\mathrm{TiS}_{2}$ 纳米晶也被报道有潜在的 电化学应用前景, 在锂离子插脱过程中只有微小 的结构变化, 同时具有较高的能量功率密度 ${ }^{71}$ 。锂 离子电池后续的研究还包括功能化的MXenes锂 离子电池的实验和理论研究, 快速冲放和高密度 储能也正在大量的科研工作中慢慢成为现实。

与当前商用化的锂离子电池石墨负极相比, 锂 金属负极具有更高的理论比容量 $\left(3860 \mathrm{mAh} \cdot \mathrm{g}^{-1}\right)$, 极低的密度 $\left(0.59 \mathrm{~g} \cdot \mathrm{cm}^{-3}\right)$ 以及最低的电化学电位 ( $-3.04 \mathrm{~V} v s$. RHE), 是最为理想的高能量密度锂电 池负极材料。然而, 由于局部非均匀电场所诱导的 锂金属不可控沉积, 枝晶状的锂不可避免地在负 极表面生长, 造成电解液的快速消耗, 甚至隔膜的 刺穿乃至电池爆炸 ${ }^{72}$ 。此外, 锂金属超高的化学活 性和体积效应也造成了锂金属负极在实际应用过 程中的一系列问题。能否采取类似锂离子存储于 二维材料层间的策略, 通过二维范德华间隙来稳 定锂金属将是一个非常有效的解决方案。这种方 法可以实现锂金属的层间限域沉积, 避免锂金属 与电解液的直接接触, 从而大大提高锂金属电极 的稳定性, 是未来层间能源存储领域的重要发展 方向之一。

\section{6 结论}

在此综述中，我们系统地阐述了二维范德华 间隙开发利用的一系列工作, 介绍了近年来学者 们在通过引入客体原子、有机分子、二维纳米片等 物质调控二维材料的范德华间隙, 从而优化二维 材料的电学、光学、量子、催化等特性的诸多尝试 与努力。二维范德华间隙的开发和利用可以综合 调控二维材料的物理化学性质, 乃至探索材料原 来不具有的新性质。然而利用插层法进行二维材 料范德华间隙的调控也存在着一些问题。首先, 层 间插入的客体物质可能引起宿主晶格的晶格畸 变, 引起应力或其他缺陷, 这些由于插层引起的晶 格畸变和缺陷可能会使散射的增加, 造成材料电 导率和热导率的下降, 引入的应力也可能会改变 
材料的能带结构和费米能级, 造成电学性质和磁 学性质的变化, 这些因素意味着层间插入的客体 物质在提高材料的一些性能的同时可能会降低材 料的其他性能。此外, 对于特定的插层剂和插层体 系, 如带电荷的离子插层, 存在插层浓度有极限或 者插入的客体不稳定等问题, 制约了层间调控的 实际效果。当前科研工作者在利用范德华间隙调 控材料物理化学特性时, 通常把目光放在优化材 料的某个特定性能上, 所使用的调控方法也较为 单一。我们认为使用多种方法共同调控材料的性 质可能是创造适合工业应用所需材料的有效方 法, 也是未来利用范德华间隙调控材料性质的发 展方向。在利用插层等方法调控材料性质的同时, 进一步应用杂原子掺杂和应变调控等方法共同优 化材料性能, 也许可以突破单一方法在调控材料 性能上的极限, 避免或弥补插层可能会造成的某 些性能下降, 从而得到性能更加优异或者更加全 面的材料。二维范德华间隙利用是调控和提升材 料性能的重要方法, 层间物质存储更是未来能源 存储领域的重要发展方向。我们相信通过研究人 员的进一步努力, 二维范德华间隙的开发利用会 真正提高二维材料的综合应用潜力和实用价值, 为将来二维材料走向工业化、走进我们的生活贡 献力量。

\section{References}

(1) Hu, Y. J.; Jin, J.; Zhang, H.; Wu, P.; Cai, C. X. Acta Phys. -Chim. Sin. 2010, 26, 2073. [胡耀娟, 金娟, 张卉, 吴萍, 蔡称心. 物理化 学学报, 2010, 26, 2073.] doi: 10.3866/PKU.WHXB20100812

(2) Zhang, P.; Ma, L. L.; Fan, F. F.; Zeng, Z.; Peng, C.; Loya, P. E.; Liu, Z.; Gong, Y. J.; Zhang, J. N.; Zhang X. X.; et al. Nat. Commun. 2014, 5, 3782. doi: $10.1038 /$ ncomms 4782

(3) Li, J. Y.; Ding, Y.; Zhang, D. W.; Zhou, P. Acta Phys. -Chim. Sin. $\mathbf{2 0 1 9}, 35,1058$. [李家意, 丁一, 张卫, 周鹏. 物理化学学报, 2019, 35, 1058.] doi: 10.3866/PKU.WHXB201812020

(4) Jiang, H. N.; Zhang, P.; Wang, X. G.; Gong, Y. J. Nano Res. 2020, doi: $10.1007 / \mathrm{s} 12274-020-3020-5$

(5) Wang, X. G.; Zhou, Z.; Zhang, P.; Zhang, S. Q.; Ma, Y.; Yang, W. W.; Wang, H.; Li, B. X.; Meng, L. J.; Jiang, H. N.; et al. Chem. Mater. 2020, 32, 2321. doi: 10.1021/acs.chemmater.9b04416

(6) Xue, Y. H.; Zhang, Q.; Wang, W. J.; Cao, H.; Yang, Q. H.; Fu, L. Adv. Energy Mater. 2017, 7, 1602684. doi: 10.1002/aenm.201602684

(7) Wang, C.; He, Q. Y.; Halim, U.; Liu, Y. Y.; Zhu, E. B.; Lin, Z. Y.; Xiao, H.; Duan, X. D.; Feng, Z. Y.; Chen, R.; et al. Nature 2018, 555, 231. doi: $10.1038 /$ nature 25774

(8) Kobayashi, K.; Yamauchi, J. Phys. Rev. B: Condens. 1995, 51,
17085. doi: 10.1103/PhysRevB.51.17085

(9) Lu, N.; Guo, H.; Wang, L.; Wu, X.; Zeng, X. C. Nanoscale 2014, 6, 4566. doi: 10.1039/C4NR00783B

(10) Conley, H. J.; Wang, B.; Ziegler, J. I.; Haglund, R. F.; Pantelides, S. T.; Bolotin, K. I. Nano Lett. 2013, 13, 3626. doi: $10.1021 / \mathrm{n} 14014748$

(11) Komsa, H. P.; Krasheninnikov, A. V. Phys. Rev. B 2013, 88, 085318. doi: $10.1103 /$ physrevb.88.085318

(12) Terrones, H.; Lopez-Urias, F.; Terrones, M. Sci. Rep. 2013, 3, 1549. doi: 10.1038/srep01549

(13) Lu, N.; Guo, H.; Li, L.; Dai, J.; Wang, L.; Mei, W. N.; Wu, X.; Zeng, X. C. Nanoscale 2014, 6, 2879. doi: 10.1039/c3nr06072a

(14) Ma, Y.; Dai, Y.; Guo, M.; Niu, C.; Huang, B. Nanoscale 2011, 3, 3883. doi: $10.1039 / \mathrm{c} 1 \mathrm{nr} 10577 \mathrm{a}$

(15) Kośmider, K.; Fernández-Rossier, J. Phys. Rev. B 2013, 87, 075451. doi: 10.1103/physrevb.87.075451

(16) Bao, W. Z.; Wan, J. Y.; Han, X. G.; Cai, X. H.; Zhu, H. L.; Kim, D. H.; Ma, D. K.; Xu, Y. L.; Munday, J. N.; Drew, H. D.; et al. Nat. Commun. 2014, 5, 4224. doi: 10.1038/ncomms5224

(17) Wan, J. Y.; Bao, W. Z.; Liu, Y.; Dai, J. Q.; Shen, F.; Zhou, L. H.; Cai, X. H.; Urban, D.; Li, Y. Y.; Jungjohann, K.; et al. Adv. Energy Mater. 2015, 5, 1401742. doi: 10.1002/aenm.201401742

(18) Kim, N.; Kim, K. S.; Jung, N.; Brus, L.; Kim, P. Nano Lett. 2011, 11, 860. doi: $10.1021 / \mathrm{n} 1104228 \mathrm{f}$

(19) Yu, Y. J.; Yang, F. Y.; Lu, X. F.; Yan, Y. J.; Cho, Y. H.; Ma, L. G.; Niu, X. H.; Kim, S.; Son, Y. W.; Feng, D. L.; et al. Nat. Nanotechnol. 2015, 10, 270. doi: 10.1038/nnano.2014.323

(20) Wu, H.; Kong, D.; Ruan, Z.; Hsu, P. C.; Wang, S.; Yu, Z.; Carney, T. J.; Hu, L.; Fan, S.; Cui, Y. Nat. Nanotechnol. 2013, 8, 421. doi: $10.1038 /$ nnano.2013.84

(21) Li, H. P.; Pan, W.; Zhang, W.; Huang, S. Y.; Wu, H. Adv. Funct. Mater. 2013, 23, 209. doi: 10.1002/adfm.201200996

(22) Motter, J. P.; Koski, K. J.; Cui, Y. Chem. Mat. 2014, 26, 2313. doi: $10.1021 / \mathrm{cm} 500242 \mathrm{~h}$

(23) Koski, K. J.; Cha, J. J.; Reed, B. W.; Wessells, C. D.; Kong, D.; Cui, Y. J. Am. Chem. Soc. 2012, 134, 7584. doi: 10.1021/ja300368x

(24) Yang, W. W.; Zhang, S. Q.; Chen, Q.; Zhang, C.; Wei, Y.; Jiang, H. N.; Lin, Y. X.; Zhao. M. T.; He, Q. Q.; Wang, X. G.; et al. Adv. Mater. 2020, 32, e2001167. doi: 10.1002/adma.202001167

(25) Friend, R. H.; Yoffe, A. D. Adv. Phys. 1987, 36, 1. doi: 10.1080/00018738700101951

(26) Kanetani, K.; Sugawara, K.; Sato, T.; Shimizu, R.; Iwaya, K.; Hitosugi, T.; Takahashi, T. Proc. Natl. Acad. Sci. U. S. A. 2012, 109 , 19610. doi: 10.1073/pnas.1208889109

(27) Bouwmeester, H. J.; van der Lee, A.; van Smaalen, S.; Wiegers, G. A. Phys. Rev. B: Condens. 1991, 43, 9431. 
doi: $10.1103 /$ physrevb.43.9431

(28) Harshman, D. R.; Mills, A. P. Phys. Rev. B: Condens. 1992, 45, 10684. doi: $10.1038 /$ nmat 4251

(29) Kumar, P.; Skomski, R.; Pushpa, R. ACS Omega. 2017, 2, 7985. doi: 10.1021/acsomega.7b01164

(30) Xie, J.; Zhang, J.; Li, S.; Grote, F.; Zhang, X.; Zhang, H.; Wang, R.; Lei, Y.; Pan, B.; Xie, Y. J. Am. Chem. Soc. 2013, 135, 17881. doi: $10.1021 / \mathrm{ja} 408329 \mathrm{q}$

(31) Qin, S.; Lei, W.; Liu, D.; Chen, Y. Sci. Rep. 2014, 4, 7582. doi: $10.1038 /$ srep07582

(32) Ye, L. J.; Chen, S. J.; Li, W. J.; Pi, M. Y.; Wu, T. L.; Zhang, D. K. J. Phys. Chem. C 2015, 119, 9560. doi: 10.1021/jp5128018

(33) Wang, C.; He, Q. Y.; Halim, U.; Liu, Y. Y.; Zhu, E. B.; Lin, Z. Y.; Xiao, H.; Duan, X. D.; Feng, Z. Y.; Cheng, R.; et al. Nature 2018, 555, 231. doi: 10.1038/nature25774

(34) Wang, M.; Koski, K. J. ACS Nano 2015, 9, 3226. doi: $10.1021 /$ acsnano. 5 b00336

(35) Wang, M. J.; Al-Dhahir, I.; Appiah, J.; Koski, K. J. Chem. Mater. 2017, 29, 1650. doi: 10.1021/acs.chemmater.6b04918

(36) Hennig, G. R. J. Chem. Phys. 1965, 43, 1201. doi: $10.1063 / 1.1696905$

(37) Yang, W. W.; Xiao, J. W.; Ma, Y.; Cui, S. Q.; Zhang, P.; Zhai, P. B.; Meng. L. J.; Wang, X. G.; Wei, Y.; Du, Z. G.; et al. Adv. Energy Mater. 2019, 9, 1803137. doi: 10.1002/aenm.201803137

(38) Wang, M.; Lahti, G.; Williams, D.; Koski, K. J. ACS Nano 2018, 12, 6163. doi: $10.1021 /$ acsnano. $8 \mathrm{~b} 02789$

(39) Gong, Y. J.; Yuan, H. T.; Wu, C. L.; Tang, P. Z.; Yang, S. Z.; Yang, A. K.; Li, G. D.; Liu, B. F.; Groep, J. V. D.; Brongersma, M. L.; et al. Nat. Nanotechnol. 2018, 13, 294. doi: 10.1038/s41565-018-0069-3

(40) He, Q.; Lin, Z.; Ding, M.; Yin, A.; Halim, U.; Wang, C.; Liu, Y.; Cheng, H. C.; Huang, Y.; Duan, X. Nano Lett. 2019, 19, 6819. doi: 10.1021/acs.nanolett.9b01898

(41) Wang, N. Z.; Tang, H. B.; Shi, M. Z.; Zhang, H.; Zhuo, W. Z.; Liu, D. Y.; Meng, F. B.; Ma, L. K.; Ying, J. J.; Zou, L. J.; et al. J. Am. Chem. Soc. 2019, 141, 17166. doi: 10.1021/jacs.9b06929

(42) Wang, H.; Lu, Z.; Kong, D.; Sun, J.; Hymel, T. M.; Cui, Y. ACS Nano 2014, 8, 4940. doi: 10.1021/nn500959v

(43) Wang, X.; Shen, X.; Wang, Z.; Yu, R.; Chen, L. ACS Nano 2014, 8, 11394. doi: $10.1021 / \mathrm{nn} 505501 \mathrm{v}$

(44) Chen, W. S.; Gu, J. J.; Liu, Q. L.; Luo, R. C.; Yao, L. L.; Sun, B. Y.; Zhang, W.; Su, H. L.; Chen, B.; Liu, P.; et al. ACS Nano 2018, 12, 308. doi: $10.1021 /$ acsnano. $7 b 06364$

(45) Meng, L. J.; Ma, Y.; Si, K. P.; Xu, S. Y.; Wang, J. L.; Gong, Y. J. Tungsten 2019, 1, 46. doi: 10.1007/s42864-019-00012-x

(46) Li, J. Y.; Zhang, Z. Y.; Cui, W.; Wang, H.; Cen, W. L.; Johnson, G.; Jiang, G. M.; Zhang, S.; Dong, F. ACS Catal. 2018, 8, 8376. doi: $10.1021 /$ acscatal.8b02459

(47) O'Farrell, E. C.; Tan, J. Y.; Yeo, Y.; Koon, G. K.; Ozyilmaz, B.; Watanabe, K.; Taniguchi, T. Phys. Rev. Lett. 2016, 117, 076603. doi: 10.1103/physrevlett.117.076603

(48) Zhao, J.; Islam, S. M.; Kontsevoi, O. Y.; Tan, G.; Stoumpos, C. C.; Chen, H.; Li, R. K.; Kanatzidis, M. G. J. Am. Chem. Soc. 2017, 139 , 6978. doi: 10.1021/jacs.7b02243

(49) Ebina, Y.; Sakai, N.; Sasaki, T. J. Phys. Chem. B 2005, 109, 17212. doi: $10.1021 / \mathrm{jp} 051823 \mathrm{j}$

(50) Hata, H.; Kubo, S.; Kobayashi, Y.; Mallouk, T. E. J. Am. Chem. Soc. 2007, 129, 3064. doi: 10.1021/ja068272a

(51) Hata, H.; Kobayashi, Y.; Salama, M.; Malek, R.; Mallouk, T. E. Chem. Mater. 2007, 19, 6588. doi: 10.1021/cm701936y

(52) Hata, H.; Kobayashi, Y.; Bojan, V.; Youngblood, W. J.; Mallouk, T. E. Nano Lett. 2008, 8, 794. doi: 10.1021/n1072571w

(53) Oshima, T.; Lu, D.; Ishitani, O.; Maeda, K. Angew. Chem. Int. Ed. 2015, 54, 2698. doi: 10.1002/ange.201411494

(54) Luo, Y.; Li, X.; Cai, X.; Zou, X.; Kang, F.; Cheng, H. M.; Liu, B. ACS Nano 2018, 12, 4565. doi: 10.1021/acsnano.8b00942

(55) Chen, Z. X.; Liu, C. B.; Liu, J.; Li J.; Xi, S. B.; Chi, X.; Xu, H. S.; Park, I. H.; Peng, X. W.; Li, X.; et al. Adv. Mater. 2020, 32, e1906437. doi: 10.1002/adma.201906437

(56) Nair, N. L.; Maniv, E.; John, C.; Doyle, S.; Orenstein, J.; Analytis, J. G. Nat. Mater. 2020, 19, 153. doi: 10.1038/s41563-019-0518-x

(57) Zhao, X. H.; Song, P.; Wang, C. C.; Anders C. Riis-Jensen; Fu, W.; Deng, Y.; Wan, D. Y.; Kang, L. X.; Ning, S. C.; Dan, J. D.; et al. Nature 2020, 581, 171. doi: 10.1038/s41586-020-2241-9

(58) Palomares, V.; Serras, P.; Villaluenga, I.; Hueso, K. B.; CarreteroGonzalez, J.; Rojo, T. Energy Environ. Sci. 2012, 5, 5884. doi: $10.1039 / \mathrm{c} 2 \mathrm{ee} 02781 \mathrm{j}$

(59) Kim, H.; Kim, J. C.; Bianchini, M.; Seo, D. H.; Rodriguez-Garcia, J.; Ceder, G. Adv. Energy Mater. 2018, 8, 1702384. doi: $10.1002 /$ aenm.201702384

(60) Song, M.; Tan, H.; Chao, D. L.; Fan, H. J. Adv. Funct. Mater. 2018, 28, 1802564. doi: 10.1002/adfm.201802564

(61) Muldoon, J.; Bucur, C. B.; Gregory, T. Chem. Rev. 2014, 114, 11683. doi: $10.1021 /$ cr500049y

(62) Zhai, P. B.; Wei, Y.; Xiao, J.; Liu, W.; Zuo, J. H.; Gu, X. K.; Yang, W. W.; Cui, S. Q.; Li, B.; Yang, S. B.; et al. Adv. Energy Mater. 2020, 10, 1903339. doi: 10.1002/aenm.201903339

(63) Chen, K.; Sun, Z. H.; Fang, R. P.; Li, F.; Cheng, H. M. Acta Phys. -Chim. Sin. 2018, 34, 377. [陈克, 孙振华, 方若翩, 李峰, 成会明. 物理化学学报, 2018, 34, 377.] doi: 10.3866/PKU.WHXB201709001

(64) Lamb, A. B. Chem. Eng. News 1942, 20, 267. doi: $10.1021 /$ cen-v020n004.p267 
(65) Rüdorff, W. J. C. Chimia 1965, 19, 489.

(66) Whittingham, M. S.; Gamble, F. R. Mater. Res. Bull. 1975, 10, 363. doi: 10.1016/0025-5408(75)90006-9

(67) Whittingham, M. S. Science 1976, 192, 1126. doi: $10.1126 /$ science. 192.4244 .1126

(68) Mizushima, K.; Jones, P. C.; Wiseman, P. J.; Goodenough, J. B. Mater. Res. Bull. 1980, 15, 783. doi: 10.1016/0025-5408(80)90012-4

(69) Mashtalir, O.; Naguib, M.; Mochalin, V. N.; Dall'Agnese, Y.; Heon, M.; Barsoum, M. W.; Gogotsi, Y. Nat. Commun. 2013, 4, 1716. doi: $10.1038 /$ ncomms 2664

(70) Eames, C.; Islam, M. S. J. Am. Chem. Soc. 2014, 136, 16270. doi: $10.1021 / \mathrm{ja} 508154 \mathrm{e}$

(71) Muller, G. A.; Cook, J. B.; Kim, H. S.; Tolbert, S. H.; Dunn, B. Nano Lett. 2015, 15, 1911. doi: 10.1021/n1504764m

(72) Liu, W.; Zhai, P. B.; Qin, S. J.; Xiao, J.; Wei, Y.; Yang, W. W.; Cui, S. Q.; Chen, Q.; Jin, C. Q.; Yang, S. B.; et al. J. Energy Chem. 2021, 56, 463. doi: 10.1016/j.jechem.2020.08.019 